

\title{
Nondeterministic Phase Semantics and the Undecidability of Boolean BI
}

Dominique Larchey-Wendling, Didier Galmiche

\section{To cite this version:}

Dominique Larchey-Wendling, Didier Galmiche. Nondeterministic Phase Semantics and the Undecidability of Boolean BI. ACM Transactions on Computational Logic, 2013, 14 (1), pp.6. 10.1145/2422085.2422091 . hal-01256956

\section{HAL Id: hal-01256956 \\ https://hal.science/hal-01256956}

Submitted on 20 Jan 2016

HAL is a multi-disciplinary open access archive for the deposit and dissemination of scientific research documents, whether they are published or not. The documents may come from teaching and research institutions in France or abroad, or from public or private research centers.
L'archive ouverte pluridisciplinaire HAL, est destinée au dépôt et à la diffusion de documents scientifiques de niveau recherche, publiés ou non, émanant des établissements d'enseignement et de recherche français ou étrangers, des laboratoires publics ou privés.

\section{(ㅇ)(1) $\$$}

Distributed under a Creative Commons Attribution - NonCommercial - NoDerivatives| 4.0 


\title{
Non-deterministic Semantics and the Undecidability of Boolean BI
}

\author{
DOMINIQUE LARCHEY-WENDLING, LORIA-CNRS, Nancy, France \\ DIDIER GALMICHE, LORIA-Université Henri Poincaré, Nancy, France
}

\begin{abstract}
We solve the open problem of the decidability of Boolean BI logic (BBI), which can be considered as the core of Separation and Spatial Logics. For this, we define a complete phase semantics for BBI and characterize it as trivial phase semantics. We deduce an embedding between trivial phase semantics for intuitionistic linear logic (ILL) and Kripke semantics for BBI. We single out the elementary fragment of ILL which is both undecidable and complete for trivial phase semantics. Thus, we obtain the undecidability of BBI.
\end{abstract}

Categories and Subject Descriptors: F.4.1 [Mathematical Logic and Formal Languages]: Mathematical Logic-Proof theory; Model Theory; Computability theory

General Terms: Theory

Additional Key Words and Phrases: Linear Logic, Boolean BI, Minsky machines, decidability

ACM Reference Format:

Larchey-Wendling, D., and Galmiche, D. 2011. The Undecidability of Boolean BI through Phase Semantics. ACM Trans. Comput. Logic 1, 1, Article 1 (June 2011), 27 pages.

DOI $=10.1145 / 0000000.0000000$ http://doi.acm.org/10.1145/0000000.0000000

\section{INTRODUCTION}

The question of the decidability of the Boolean version of the logic of Bunched Implications (denoted $\mathrm{Bl}$ ) was a longstanding open problem. BI itself was proved decidable by Galmiche et al. [Galmiche et al. 2005] and Boolean BI was naively thought "simpler" than BI until a faithful embedding from $\mathrm{BI}$ into Boolean $\mathrm{BI}$ was discovered [Larchey-Wendling and Galmiche 2009]. Independently, Brotherston and Kanovich [Brotherston and Kanovich 2010] on the one hand and Larchey-Wendling and Galmiche [Larchey-Wendling and Galmiche 2010] on the other hand recently solved the issue by different techniques: the former by focusing mainly on the links between Boolean BI and Separation Logic, the later by establishing semantic links between Intuitionistic Linear Logic (ILL) and Boolean BI. This paper is an enriched and self-contained version of the results and proofs of [Larchey-Wendling and Galmiche 2010].

The logic BI of bunched implications [O'Hearn and Pym 1999] is a sub-structural logic which freely combines additive connectives $\wedge, \vee, \rightarrow$ and multiplicative connectives $*, *$. In $\mathrm{Bl}$, both the multiplicatives and the additives behave intuitionistically. From its inception, $\mathrm{BI}$ was given a nice bunched sequent proof-system enjoying cut-elimination [Pym 2002]. Later, [Galmiche et al. 2005] gave $\mathrm{BI}$ a sound and complete labeled tableaux system from which decidability was derived. The $\operatorname{logic} \mathrm{BI}$ is sometimes called intuitionistic $\mathrm{BI}$ to distinguish it with other variants where either the multiplicatives or the additives include a negation and thus behave classically.

From a proof-theoretical perspective, Boolean $\mathrm{BI}$ (or simply BBI) can be considered as the first investigated variant of $\mathrm{BI}$ which contained a negation: BBI combines intuitionistic multiplicatives with Boolean additives. This focus on $\mathrm{BBI}$ is the consequence of the natural links between $\mathrm{BBI}$ and separation or spatial logics. Indeed, for instance, the pure part of separation logic is essentially obtained by considering a particular model of BBI, based on a (partial) monoid of heaps [Ishtiaq and O'Hearn 2001] (see [Larchey-Wendling and Galmiche 2009] for a more general discussion on these links). The Hilbert proof-system of BBI was proved complete w.r.t. relational (or non-deterministic) Kripke semantics [Galmiche and Larchey-Wendling 2006]. However, the proof-theory of BBI was rather poorly developed because it was difficult to conceive how the bunched sequent calculus of (intuitionistic) BI could be extended to BBI without losing key properties such as e.g. cut-elimination.

Two main families of results emerged giving a contrasted view of its proof-theory. On the one hand, [Brotherston 2010] adapted the Display proof-system of Classical BI to BBI, circumventing the difficulty of the multiplicatives of BBI lacking a negation. This system was proved sound and 
complete w.r.t. relational Kripke semantics. Cut-elimination was also derived but, despite the expectations of Brotherston, no decidability result followed. On the other hand, [Larchey-Wendling and Galmiche 2009] proposed a labeled tableaux proof-system for (partial monoidal) BBI and by the study of the relations between the proof-search generated counter-models of $\mathrm{BI}$ and BBI, showed that (intuitionistic) BI could be faithfully embedded into BBI. This result, at first counter-intuitive, hinted that $\mathrm{BBI}$, originally thought simpler than $\mathrm{BI}$, could in fact be much more difficult to decide.

In this paper, we consider models of BBI belonging to different classes:

ND. The class of non-deterministic monoids;

PD. The class of partial (deterministic) monoids;

TD. The class of total (deterministic) monoids;

HM. The class of heaps monoids (i.e. separation logic models);

FM. The class of free monoids.

Generally, each class of models defines a different notion of (universal Kripke) validity on the formulae of $\mathrm{BBI}$. For instance, we recall the result that the set $\mathrm{BBI}_{\mathrm{ND}}$ of $\mathrm{BBI}$-formulae valid in every non-deterministic monoid is strictly included in the set $\mathrm{BB} \mathrm{I}_{\mathrm{PD}}$ of $\mathrm{BBI}$-formulae valid in every partial deterministic monoid [Larchey-Wendling and Galmiche 2010]. The classification of these classes of models with respect to BBI Kripke validity is not finished though.

The principal result of this paper is the undecidability of universal validity in $\mathrm{BBI}$, whichever class of models is chosen amongst ND, PD, TD, HM and FM. Although these classes of models generally define different notions of universal validity for the whole $\mathrm{BBI}$, we have identified a fragment of $\mathrm{BBI}$ on which these semantics collapse to one. This fragment is the direct image of the elementary fragment of ILL (denoted elLL) by an embedding of ILL into BBI. This elementary fragment is different from the minimal fragment of Boolean BI identified in [Brotherston and Kanovich 2010] but has similar properties. In our case, undecidability is obtained by the following steps:

- we show that the embedding of elLL into BBI is faithful for trivial phase semantics;

- we show that the elLL fragment is complete for trivial phase semantics, whichever class of models is chosen amongst ND, PD, TD, HM and FM;

- we show how to encode the computations of two counter Minsky machines in elLL.

As a consequence, we derive the undecidability of the elLL fragment, from which we deduce the undecidability of BBI. We complete the pictures with additional results of undecidability on the models based on the free monoid $\mathbb{N} \times \mathbb{N}$ and the models based on the partial monoid $\mathbb{P}_{\mathrm{f}}(\mathbb{N}$ ) (which is also the simplest heap monoid). This last result is obtained using bisimulation techniques.

Compared to the original LICS'10 paper [Larchey-Wendling and Galmiche 2010], this paper contains a more extensive study of the semantics of the elLL fragment with completeness results for various classes of models and the adaptation of our undecidability result of BBI to heaps models (i.e. to Separation Logic) using a bisimulation between free monoids and heap monoids.

\section{CLASSES OF NON-DETERMINISTIC MONOIDS}

In this section, we define the algebraic notion of non-deterministic (commutative) monoid. We denote algebraic structure by $\mathcal{M}, \mathcal{N}, \ldots$ classes of structures by $\mathrm{C}, \mathrm{D}, \ldots$ sets by $\mathrm{X}, \mathrm{Y}, \ldots$ elements by $x$, $y, \ldots$ and well known constructs like the powerset by $\mathbb{P}(\mathbf{X})$ or the set of (finite) multisets by $\mathbb{M}_{\mathrm{f}}(\mathrm{X})$. The symbol $\mathbb{N}=\{0,1,2, \ldots\}$ denotes the set of natural numbers. The symbol $\emptyset$ is used either to denote the empty set, the empty multiset or the empty class.

\subsection{Non-deterministic monoids}

Let us consider a set $M$ and its powerset $\mathbb{P}(M)$, i.e. the set of subsets of $M$. A composition is a binary function $\circ: \mathrm{M} \times \mathrm{M} \longrightarrow \mathbb{P}(\mathrm{M})$ which naturally extended to a binary operator on $\mathbb{P}(\mathrm{M})$ by

$$
\mathrm{X} \circ \mathrm{Y}=\bigcup\{x \circ y \mid x \in \mathrm{X} \text { and } y \in \mathrm{Y}\}
$$


for any subsets $\mathrm{X}, \mathrm{Y}$ of $\mathrm{M}$. Using this extension, we can view an element $m$ of $\mathrm{M}$ as the singleton set $\{m\}$ and derive equations like $m \circ \mathbf{X}=\{m\} \circ \mathbf{X}$ and $a \circ b=\{a\} \circ\{b\}$ by a slight abuse of notation.

Definition 2.1. A non-deterministic (or relational) monoid is a triple $(\mathrm{M}, \circ, \epsilon)$ where $\mathrm{M}$ is a set, $\epsilon \in \mathrm{M}$ is the neutral element and $\circ: \mathrm{M} \times \mathrm{M} \longrightarrow \mathbb{P}(\mathrm{M})$ is the composition operator. In addition, the following axioms are mandatory:

$$
\begin{array}{ll}
\forall a \in \mathrm{M}, \epsilon \circ a=\{a\} & \text { (neutrality) } \\
\forall a, b \in \mathrm{M}, a \circ b=b \circ a & \text { (commutativity) } \\
\forall a, b, c \in \mathrm{M}, a \circ(b \circ c)=(a \circ b) \circ c & \text { (associativity) }
\end{array}
$$

The class of non-deterministic monoids is denoted ND.

Associativity should be understood using the extension of $\circ$ to $\mathbb{P}(\mathrm{M})$ as defined by Equation (1). The extension of $\circ$ to $\mathbb{P}(M)$ induces a commutative monoidal structure with unit element $\{\epsilon\}$ on $\mathbb{P}(M)$. As a consequence, the structure $(\mathbb{P}(\mathrm{M}), \circ,\{\epsilon\})$ is a (usual) commutative monoid.

The term non-deterministic was introduced in [Galmiche and Larchey-Wendling 2006] in order to emphasize the fact that the composition $a \circ b$ may yield not only one but an arbitrary number of results including the possible incompatibility of $a$ and $b$ in which case $a \circ b=\emptyset$. If $(\mathrm{M},+, 0)$ is a (usual) commutative monoid then, defining $a \circ b=\{a+b\}$ and $\epsilon=0$ induces a non-deterministic monoid (M,,$\epsilon)$. Using the bijection $x \mapsto\{x\}$ mapping elements of $M$ to singletons in $\mathbb{P}(\mathrm{M})$, we can view (usual) commutative monoids as a particular case of non-deterministic monoids (later called total deterministic monoids). Partial monoids can also be represented using the empty set $\emptyset$ as the result of undefined compositions (see section 2.2).

The term relational is sometimes used because the map $\circ: \mathrm{M} \times \mathrm{M} \longrightarrow \mathbb{P}(\mathrm{M})$ can equivalently be understood as a ternary relation $-\circ-\ni-: \mathrm{M} \times \mathrm{M} \times \mathrm{M} \longrightarrow\{0,1\}$ through the Curry-Howard isomorphism and the axioms correspond to those of an internal monoid in the category of relations [Ghilardi and Meloni 1990]. The two presentations are equivalent but we rather use the monoidal presentation in this paper because it better suits the context and habits of phase semantics and Kripke semantics.

\subsection{Sub-classes of non-deterministic monoids}

Definition 2.2. Let $(\mathrm{M}, \circ, \epsilon)$ be a non-deterministic monoid. It is a partial deterministic monoid if for all $x, y \in \mathrm{M}$, the composition $x \circ y$ is either empty or a singleton. It is a total deterministic monoid if for all $x, y \in \mathrm{M}$, the composition $x \circ y$ is a singleton. We use PD (resp. TD) to represent the sub-class of partial deterministic (resp. total deterministic) monoids.

The reader may have noticed that total deterministic monoids (of class TD) exactly correspond to those non-deterministic monoids derived from usual commutative monoids via the map $x \mapsto\{x\}$ because the composition $\circ$ is a functional relation in this case (exactly one image for each pair of parameters).

Let us give an example of non-deterministic monoid which shows that the class ND contains structures that have properties which are fundamentally different from those of partial or total monoids. The non-deterministic monoid $(\{\epsilon, \mathrm{x}, \mathrm{y}\}, \circ, \epsilon)$ built over this 3 elements set and defined by the following composition operator:

\begin{tabular}{c|ccc}
$\circ$ & $\epsilon$ & $\mathrm{x}$ & $\mathrm{y}$ \\
\hline$\epsilon$ & $\{\epsilon\}$ & $\{\mathrm{x}\}$ & $\{\mathrm{y}\}$ \\
$\mathrm{x}$ & $\{\mathrm{x}\}$ & $\{\epsilon, \mathrm{y}\}$ & $\{\mathrm{y}\}$ \\
$\mathrm{y}$ & $\{\mathrm{y}\}$ & $\{\mathrm{y}\}$ & $\{\mathrm{y}\}$
\end{tabular}

is an example of such non-deterministic monoid. It is a witness that PD is a proper sub-class of ND. But also, we see that in this monoid, $x$ is both self inverse $(\epsilon \in x \circ x)$ and this same composition yields the absorbing element $(\mathrm{y} \in x \circ x)$. In Section 6.1, we will see that BBI is able to witness the difference between the class ND and the class PD. 
A typical sub-class of partial deterministic monoids is obtained by considering disjoint union over the powerset. Given a set $X$, consider the partial deterministic monoid $(\mathbb{P}(X), \uplus, \emptyset)$ where $\emptyset$ is the empty subset of $X$ and $\uplus$ is defined for $A, B \subseteq X$ by

$$
A \uplus B= \begin{cases}\emptyset & \text { when } A \cap B \neq \emptyset \\ \{A \cup B\} & \text { when } A \cap B=\emptyset\end{cases}
$$

One could even restrict to finite subsets of $X$ by considering the partial monoid $\left(\mathbb{P}_{f}(X), \uplus, \emptyset\right)$ where $\mathbb{P}_{f}(X)$ is the set of finite subsets of $X$.

A (more general) sub-class of partial deterministic monoids is of particular importance to Separation Logic [Ishtiaq and O'Hearn 2001]. Given an (infinite) set L of locations and a (non-empty) set $\mathrm{V}$ of values, a heap is a partial function from locations to values defined only on a finite number of locations. We define

$$
\mathbb{H}_{\mathrm{L}, \mathrm{V}}=\left\{h: \mathrm{L} \longrightarrow_{\mathrm{f}} \mathrm{V} \mid \operatorname{def}(h) \text { is finite }\right\} \quad \text { where } \operatorname{def}(h)=\{l \in \mathrm{L} \mid h(l) \text { is defined }\}
$$

so $\operatorname{def}(h)$ is the (finite) set of locations on which $h$ is defined. The binary composition $s \uplus t$ of two heaps $s, t \in \mathbb{H}_{\mathrm{L}, \mathrm{V}}$ is defined by

$$
s \uplus t=\left\{\begin{array}{ll}
\emptyset & \text { when } \operatorname{def}(s) \cap \operatorname{def}(t) \neq \emptyset \\
\{r\} & \text { when } \operatorname{def}(s) \cap \operatorname{def}(t)=\emptyset
\end{array} \quad \text { with } \operatorname{graph}(r)=\operatorname{graph}(s) \cup \operatorname{graph}(t)\right.
$$

The heap defined nowhere (i.e. with an empty graph) is denoted $\varnothing$. The heap monoid ( $\left.\mathbb{H}_{L, V}, \uplus, \varnothing\right)$ is a partial deterministic monoid of class PD. We point out that when $\mathrm{V}=\{*\}$ is a singleton set, then the heap monoid $\left(\mathbb{H}_{\mathrm{L},\{*\}}, \uplus, \varnothing\right)$ is isomorphic to finite powerset monoid $\left(\mathbb{P}_{\mathrm{f}}(\mathrm{L}), \uplus, \emptyset\right)$. Hence, the class of heap monoids contains (an isomorphic copy of) the class of finite powersets. The class of heap monoids is denoted HM:

$$
\mathrm{HM}=\left\{\left(\mathbb{H}_{\mathrm{L}, \mathrm{V}}, \uplus, \varnothing\right) \mid \mathrm{L} \text { is infinite and } \mathrm{V} \text { is not empty }\right\}
$$

It is obviously a sub-class of PD. Since for any non-empty heap $h$ we have $h \uplus h=\emptyset$ (but $\varnothing \uplus \varnothing=\{\varnothing\}$ ), it is clear that no heap monoid $\mathbb{H}_{\mathrm{L}, \mathrm{V}}$ is a total deterministic monoid (because neither $\mathrm{L}$ nor $\mathrm{V}$ is empty). Hence, HM and TD are two disjoint sub-classes of PD.

Another important sub-class of non-deterministic monoids is the class of FM of free (commutative) monoids $\left(\mathbb{M}_{f}(X), \star, \pi\right)$ where $X$ is a set, $\mathbb{M}_{f}(X)$ denotes the set of (finite) multisets of elements of $\mathrm{X}$, and $\star$ (resp. $\pi$ ) denotes multiset addition (resp. the empty multiset). When $\mathrm{X}$ is not empty, $\mathbb{M}_{\mathrm{f}}(\mathrm{X})$ contains an element $x \neq \pi$ and in this case, $x \star x \neq\{x\}$. Since there are total deterministic monoids satisfying the axiom $x \star x=\{x\}$ (for example lattices), we deduce that FM is a proper sub-class of TD.

Proposition 2.3. $\mathrm{FM} \subsetneq \mathrm{TD} \subsetneq \mathrm{PD} \subsetneq \mathrm{ND}, \mathrm{HM} \subsetneq \mathrm{PD}$ and $\mathrm{HM} \cap \mathrm{TD}=\emptyset$.

\section{SEQUENT CALCULUS AND PHASE SEMANTICS FOR ILL}

Linear Logic and Intuitionistic Linear Logic (denoted ILL) are well know sub-structural logics introduced by Girard in [Girard 1987] to better study the impact of structural rules on the prooftheoretical as well as semantical properties of logics. The reader can consult [Troelstra 1992] for an overview on those topics.

The formulae of ILL are defined by the following grammar:

$$
A::=v|c| ! A \mid A \circledast A \quad \text { with } v \in \operatorname{Var}, c \in\{1, \top, \perp\}^{1} \text { and } \circledast \in\{\otimes,-, \&, \oplus\}
$$

A sequent is a pair denoted $\Gamma \vdash A$ where $\Gamma$ is a (finite) multiset of formulae and $A$ is a single formula. The sequent calculus S-ILL (see Figure 1) is provided for ILL and the set of derivable sequents is

\footnotetext{
${ }^{1}$ Sometimes the neutral of $\oplus$ is denoted 0, but we favor $\perp$ as in [Troelstra 1992].
} 


$$
\begin{aligned}
& \frac{}{A \vdash A}\langle\mathrm{id}\rangle \quad \frac{}{\Gamma, \perp \vdash A}\left\langle\perp_{L}\right\rangle \quad \frac{}{\Gamma \vdash \mathrm{T}}\left\langle\mathrm{T}_{R}\right\rangle \quad \frac{}{\vdash 1}\left\langle 1_{R}\right\rangle \quad \frac{\Gamma \vdash A \quad A, \Delta \vdash B}{\Gamma, \Delta \vdash B}\langle\text { cut }\rangle \\
& \frac{\Gamma, A \vdash B}{\Gamma, ! A \vdash B}\left\langle!_{L}\right\rangle \quad \frac{! \Gamma \vdash B}{! \Gamma \vdash ! B}\left\langle!_{R}\right\rangle \quad \frac{\Gamma \vdash B}{\Gamma, ! A \vdash B}\langle\mathrm{w}\rangle \quad \frac{\Gamma, ! A, ! A \vdash B}{\Gamma, ! A \vdash B}\langle\mathrm{c}\rangle \quad \frac{\Gamma \vdash A}{\Gamma, 1 \vdash A}\left\langle 1_{L}\right\rangle \\
& \frac{\Gamma, A \vdash C}{\Gamma, A \& B \vdash C}\left\langle \&_{L}^{1}\right\rangle \quad \frac{\Gamma, B \vdash C}{\Gamma, A \& B \vdash C}\left\langle \&_{L}^{2}\right\rangle \quad \frac{\Gamma \vdash A \quad \Gamma \vdash B}{\Gamma \vdash A \& B}\left\langle \&_{R}\right\rangle \\
& \frac{\Gamma, A \vdash C \quad \Gamma, B \vdash C}{\Gamma, A \oplus B \vdash C}\left\langle\oplus_{L}\right\rangle \quad \frac{\Gamma \vdash A}{\Gamma \vdash A \oplus B}\left\langle\oplus_{R}^{1}\right\rangle \quad \frac{\Gamma \vdash B}{\Gamma \vdash A \oplus B}\left\langle\oplus_{R}^{2}\right\rangle \\
& \frac{\Gamma, A, B \vdash C}{\Gamma, A \otimes B \vdash C}\left\langle\otimes_{L}\right\rangle \quad \frac{\Gamma \vdash A \Delta \vdash B}{\Gamma, \Delta \vdash A \otimes B}\left\langle\otimes_{R}\right\rangle \quad \frac{\Gamma \vdash A \quad \Delta, B \vdash C}{\Gamma, \Delta, A \multimap B \vdash C}\left\langle\multimap_{L}\right\rangle \quad \frac{\Gamma, A \vdash B}{\Gamma \vdash A \multimap B}\left\langle\multimap_{R}\right\rangle
\end{aligned}
$$

Fig. 1. Sequent calculus S-ILL for ILL

the least set closed under its rules. Notice that $\Gamma, \Delta$ denote multisets of formulae and $A, B, C$ denote formulae. In rule $\left\langle!_{R}\right\rangle, ! \Gamma$ denotes the multiset $! \Gamma=! A_{1}, \ldots, ! A_{k}$ if $\Gamma=A_{1}, \ldots, A_{k}{ }^{2}$

The notion of sequent calculus proof is defined as usual: an ordered tree where each node together with its sons correspond to an instance of one of the rules of S-ILL. Hence, a sequent is derivable if and only if there exists a proof of it in S-ILL. By historical definition of ILL [Girard 1987], the sequents which are provable in S-ILL are exactly the valid sequents of ILL, and a formula $A$ of ILL is valid if $r A$ is a valid sequent.

\subsection{Non-deterministic phase spaces for ILL}

We extend the notion of intuitionistic phase space [Girard 1987] to non-deterministic monoids and show that this semantic interpretation is sound and complete w.r.t. S-ILL, and thus equivalent to the original notion (see Corollary 3.6).

Definition 3.1. A non-deterministic (intuitionistic) phase space is given by a non-deterministic monoid $\mathcal{M}=(\mathrm{M}, \circ, \epsilon)$ together with a stable closure operator $(\cdot)^{\diamond}: \mathbb{P}(\mathrm{M}) \longrightarrow \mathbb{P}(\mathrm{M})$ and a sub-monoid $\mathrm{K}$ included in $\mathrm{J}=\left\{x \in \mathrm{M} \mid x \in\{\epsilon\}^{\diamond} \cap(x \circ x)^{\triangleright}\right\}$.

— the closure property corresponds to the condition

$$
X \subseteq Y^{\diamond} \text { iff } X^{\diamond} \subseteq Y^{\diamond} \text { for any } X, Y \in \mathbb{P}(M)
$$

We recall that the monoidal composition $\circ$ is naturally extended to $\mathbb{P}(\mathrm{M})$ by Equation (1) providing a (commutative) monoidal structure on $\mathbb{P}(\mathrm{M})$ with unit $\{\epsilon\}$. A subset $\mathrm{X}$ of $\mathrm{M}$ is $(\cdot)^{\diamond}$-closed (or simply closed when the closure operator is obvious from the context) if $\mathrm{X}^{\diamond}=\mathrm{X}$ or equivalently $\mathrm{X}^{\diamond} \subseteq \mathrm{X}$. The set of closed subsets is denoted $\mathcal{M}^{\diamond}=\left\{\mathrm{X} \in \mathbb{P}(\mathrm{M}) \mid \mathrm{X}^{\diamond}=\mathrm{X}\right\}$, not to be confused with $\mathrm{M}^{\diamond}$ where $M$ is viewed as the (total) subset of $M$ (and in this case, $M^{\circ}=M$ ). Any intersection of closed subsets is a closed subset and thus $\mathcal{M}^{\diamond}$ is invariant under arbitrary intersections, inducing a complete lattice structure on $\left(\mathcal{M}^{\diamond}, \subseteq\right)$.

- the stability property ${ }^{3}$ corresponds to the condition

$$
\mathrm{X}^{\diamond} \circ \mathrm{Y}^{\diamond} \subseteq(\mathrm{X} \circ \mathrm{Y})^{\diamond} \text { for any } \mathrm{X}, \mathrm{Y} \in \mathbb{P}(\mathrm{M})
$$

\footnotetext{
${ }^{2}$ Notice that when multisets are considered as syntactic objects, it is usual to denote the composition of multisets by a comma whereas when they are considered as semantic objects, then the denotation of the composition operator (and the neutral element) might differ. For example, we will use $\star$ and $\pi$ in this paper.

${ }^{3}$ A stable closure is a quantic nucleus in quantale theory [Yetter 1990]. The "stability" property itself seems to have no well established terminology.
} 
Let $\multimap$ be the adjoint of $\circ$ as a binary operator on $\mathbb{P}(\mathrm{M})$. It is defined by $\mathrm{X} \multimap \mathrm{Y}=\{k \in \mathrm{M} \mid k \circ \mathrm{X} \subseteq \mathrm{Y}\}$ for any $X, Y \in \mathbb{P}(M)$. In the lattice $(\mathbb{P}(M), \subseteq)$, the operator $\multimap$ is contra-variant in its first parameter and co-variant in its second and the following adjoint property holds

$$
Z \subseteq X \multimap Y \text { iff } Z \circ X \subseteq Y \quad \text { for any } X, Y, Z \in \mathbb{P}(M)
$$

By stability of the closure operator $(\cdot)^{\diamond}$, the subset $X \multimap Y$ is closed as soon as $Y$ is closed and $\mathrm{X} \multimap \mathrm{Y}^{\diamond}=\mathrm{X}^{\diamond} \multimap \mathrm{Y}^{\diamond}$ holds for any $\mathrm{X}, \mathrm{Y} \in \mathbb{P}(\mathrm{M})$.

- the set $\mathrm{K}$ is a given sub-monoid of $\mathcal{M}$ included in $\mathrm{J}$, i.e. $\mathrm{K}$ verifies both

$$
\epsilon \in \mathrm{K} \subseteq \mathrm{J} \quad \text { and } \mathrm{K} \circ \mathrm{K} \subseteq \mathrm{K}
$$

We see that we have a (quite direct) generalization of the usual notion of phase space in the case where the monoid is neither supposed to be total nor deterministic. In the particular case of total deterministic monoids, we recover the usual notion of phase space.

The interpretation of ILL connectives is done in the following way. Given an interpretation of logical variables as closed subsets $[\cdot]: \operatorname{Var} \longrightarrow \mathcal{M}^{\diamond}$, this interpretation is extended to all the formulae of ILL by structural induction as follows:

$$
\begin{aligned}
& \llbracket \perp \rrbracket=\emptyset \triangleright \\
& \llbracket \top \rrbracket=M \\
& \llbracket 1 \rrbracket=\{\epsilon\}^{\diamond} \\
& \llbracket ! A \rrbracket=(\mathrm{K} \cap \llbracket A \rrbracket) \\
& \llbracket A \oplus B \rrbracket=(\llbracket A \rrbracket \cup \llbracket B \rrbracket)^{\diamond} \\
& \llbracket A \& B \rrbracket=\llbracket A \rrbracket \cap \llbracket B \rrbracket \\
& \llbracket A \otimes B \rrbracket=(\llbracket A \rrbracket \circ \llbracket B \rrbracket \rrbracket)^{\diamond} \\
& \llbracket A \multimap B \rrbracket=\llbracket A \rrbracket \multimap \llbracket B \rrbracket
\end{aligned}
$$

When the interpretation is done in a total deterministic monoid, we obtain exactly the same value for $\llbracket A \rrbracket$ as in the usual phase semantics interpretation.

Definition 3.2. A sequent $A_{1}, \ldots, A_{k} \vdash B$ of ILL is valid in the interpretation $[\cdot]$ if the inclusion $\llbracket A_{1} \rrbracket \circ \cdots \circ \llbracket A_{k} \rrbracket \subseteq \llbracket B \rrbracket$ holds.

We recall the soundness theorem which states that provability in S-ILL entails semantic validity in non-deterministic intuitionistic phase semantics.

Theorem 3.3 (Soundness of Phase Semantics). If the sequent $A_{1}, \ldots, A_{k} \vdash B$ has a proof in S-ILL then the inclusion relation $\llbracket A_{1} \rrbracket \circ \cdots \circ \llbracket A_{k} \rrbracket \subseteq \llbracket B \rrbracket$ holds.

Proof. The proof of this theorem can be done directly by generalizing the soundness proof of usual phase semantics [Girard 1987], or else, as done in Appendix A by using the algebraic semantic characterization of ILL of [Troelstra 1992].

Definition 3.4. We denote by $\mathrm{ILL}_{p}$ the set of sequents which have a proof in S-ILL. We denote by ILL $L_{X}$ the set of sequents which are valid in every non-deterministic phase semantic interpretation where the base monoid is of the class $\mathrm{X}$.

In this paper, the class $X$ ranges over the following set of classes $\{\mathrm{ND}, \mathrm{PD}, \mathrm{TD}, \mathrm{HM}, \mathrm{FM}\}$. Let us consider the following inclusion sequence:

$$
\mathrm{ILL}_{p} \subseteq \mathrm{ILL}_{\mathrm{ND}} \subseteq \mathrm{ILL} \mathrm{PD} \subseteq \mathrm{ILL} \mathrm{TD} \subseteq \mathrm{ILL}_{\mathrm{FM}} \subseteq \mathrm{ILL}_{p}
$$

The first inclusion ILL $\mathrm{IL}_{p} \subseteq \mathrm{ILL}$ ND is given by Theorem 3.3. The following inclusions ILL $\mathrm{ND} \subseteq \mathrm{ILL}$ PD $\subseteq$ $\mathrm{ILL}_{\mathrm{TD}} \subseteq \mathrm{ILL}_{\mathrm{FM}}$ are obvious consequences of the inclusions $\mathrm{FM} \subseteq \mathrm{TD} \subseteq \mathrm{PD} \subseteq \mathrm{ND}$ between classes of non-deterministic monoids. The last inclusion ILLFM $\subseteq \mathrm{ILL} p$ is just a reformulation of the completeness of the phase semantics w.r.t. S-ILL:

Theorem 3.5 (Completeness of Phase Semantics). If the sequent $\Gamma \vdash A$ is valid in every free monoidal phase semantic interpretation $\left(\mathrm{M}, \circ, \epsilon,(\cdot)^{\diamond}, \mathrm{K},[[\cdot])\right.$ (i.e. with $(\mathrm{M}, \circ, \epsilon)$ of the class $\left.\mathrm{FM}\right)$, then $\Gamma \vdash A$ has a proof in S-ILL. 
Proof. The proof is based on a very nice semantic argument first introduced by Okada [Okada 2002]. Nevertheless, as its understanding is not really critical to the developments of this paper, it is postponed to Appendix B.

COROLLARY 3.6. ILL $\mathrm{IL}_{p}=\mathrm{ILL} \mathrm{ND}=\mathrm{ILL} \mathrm{PD}_{\mathrm{PD}}=\mathrm{ILL} \mathrm{TD}=\mathrm{IL} \mathrm{LM}_{\mathrm{FM}}$ and non-deterministic phase semantics is both sound and complete w.r.t. S-ILL.

Proof. With Theorem 3.5, we have closed the circular inclusion sequence (2). In particular $\mathrm{ILL}_{p}=\mathrm{ILL} \mathrm{ND}$.

Remark: we let the question of determining whether ILL $\mathrm{LHM}_{\mathrm{HM}}=\mathrm{ILL} \mathrm{L}_{p}$ or else $\mathrm{ILL}_{\mathrm{HM}} \nsubseteq \mathrm{ILL} \mathrm{L}_{p}$ as an open question.

\subsection{Trivial phase semantics for ILL}

In this section, we define trivial phase semantics which is a particular case of phase semantics where the choice of the least closure operator is mandatory.

Definition 3.7. Given a non-deterministic monoid $\mathcal{M}=(\mathrm{M}, \circ, \epsilon)$, the trivial phase space is defined by taking the identity map on $\mathbb{P}(M)$ as closure operator (i.e. for all $X \in \mathbb{P}(M), X^{\diamond}=X$ ) and by taking $\mathrm{K}=\{\epsilon\}$.

It is clear that the identity on $\mathbb{P}(\mathrm{M})$ is both a closure and stable. Obviously also, $\mathrm{K}=\{\epsilon\}$ verifies the conditions $\epsilon \in \mathrm{K} \subseteq \mathrm{J}$ and $\mathrm{K} \circ \mathrm{K} \subseteq \mathrm{K}$. ${ }^{4}$ In a trivial phase space, every subset of $\mathrm{M}$ is closed and thus $\mathcal{M}^{\diamond}=\mathbb{P}(\mathrm{M})$. The interpretation of ILL connectives simplifies to:

$$
\begin{aligned}
& \llbracket \perp \rrbracket=\emptyset \\
& \llbracket \top \rrbracket=\mathrm{M} \\
& \llbracket 1 \rrbracket=\{\epsilon\} \\
& \llbracket A \oplus B \rrbracket=\llbracket A \rrbracket \cup \llbracket B \rrbracket \\
& \llbracket A \& B \rrbracket=\llbracket A \rrbracket \cap \llbracket B \rrbracket \\
& \llbracket ! A \rrbracket=\{\epsilon\} \cap \llbracket A \rrbracket \\
& \llbracket A \otimes B \rrbracket=\llbracket A \rrbracket \circ \llbracket B \rrbracket \\
& \llbracket A \multimap B \rrbracket=\llbracket A \rrbracket \multimap \llbracket B \rrbracket
\end{aligned}
$$

Trivial phase semantics is not complete for the whole ILL. Indeed, the additive operators $\oplus$ and $\&$ become distributive over each other in trivial phase semantics. This is not the case in (general) phase semantics. In particular, the formula $A \&(B \oplus C) \multimap(A \& B) \oplus(A \& C)$ is valid in trivial phase semantics but has no proof in S-ILL.

However not complete for the whole ILL, we are now going to a introduce the elementary fragment of ILL which is complete for trivial phase semantics and nevertheless sufficiently expressive to be able to encode computations of Minsky machines.

\section{ELEMENTARY INTUITIONISTIC LINEAR LOGIC AND TRIVIAL PHASE SEMANTICS}

We define and characterize elementary ILL (denoted eILL), an extension of the fragment S-IMELL ${ }_{0}^{-}$ of ILL [de Groote et al. 2004]. We provide a simple goal-directed proof system, denoted G-elLL, which is itself an extension of the goal-directed proof system of s-IMELL -0 , obtained by the addition of a new additive rule. Then we show that the proof system G-elLL and trivial phase semantics are both sound and complete w.r.t. the fragment elLL. We also show that validity in trivial phase semantics does not depend on a particular class of models on the elementary fragment: all classes among $\{\mathrm{ND}, \mathrm{PD}, \mathrm{TD}, \mathrm{FM}\}$ define the same set of (universally) valid elementary sequents.

\subsection{The elLL fragment of ILL}

Definition 4.1. A formula of ILL is $(\multimap, \&)$-elementary if it is of the form $u \multimap v,(u \multimap v) \multimap w$, $u \multimap(v \multimap w)$ or $(u \& v) \multimap w$ where $u, v$ and $w$ are logical variables in Var. The sequents of the fragment elLL are those of the form $! \Sigma, \Gamma \vdash c$ where $\Gamma$ is a multiset of variables, $c$ is a variable and $\Sigma$ is a multiset of $(\multimap, \&)$-elementary formulae.

${ }^{4}$ In fact, there is no other possible choice for $\mathrm{K}$ because $\mathrm{J}=\left\{x \in \mathrm{M} \mid x \in\{\epsilon\}^{\diamond} \cap(x \circ x)^{\diamond}\right\}=\{\epsilon\}$ when $(\cdot)^{\diamond}$ is the identity map on $\mathbb{P}(\mathrm{M})$. 


$$
\begin{gathered}
\frac{! \Sigma, \Gamma \vdash u}{! \Sigma, u \vdash u}\langle\mathrm{Ax}\rangle \quad \frac{!}{! \Sigma, \Gamma \vdash v} u \multimap v \in \Sigma \quad \frac{! \Sigma, \Gamma, u \vdash v}{! \Sigma, \Gamma \vdash w}(u \multimap v) \multimap w \in \Sigma \\
\frac{! \Sigma, \Gamma \vdash u \quad ! \Sigma, \Delta \vdash v}{! \Sigma, \Gamma, \Delta \vdash w} u \multimap(v \multimap w) \in \Sigma \quad \frac{! \Sigma, \Gamma \vdash u \quad ! \Sigma, \Gamma \vdash v}{! \Sigma, \Gamma \vdash w}(u \& v) \multimap w \in \Sigma
\end{gathered}
$$

Fig. 2. G-elLL: a goal-directed sequent calculus for elLL

From this definition, it is obvious that membership in the fragment elLL is a recursive property. Compared to $\mathrm{S}-\mathrm{IMELL}_{0}^{-}$, the only new form is $(u \& v) \multimap w$. The validity of sequents in elLL can be established using the proof system S-ILL but we rather provide an alternative goal-directed proof system called G-elLL in Figure 2. Apart for the axiom rule $\langle A x\rangle$, each other rule $\langle\multimap\rangle,\langle(-\circ)-\rangle$, $\langle\multimap(-)\rangle$ or $\langle(\&) \multimap\rangle$ is named according to the form of its side condition. Compared to $\mathrm{S}-\mathrm{IMELL}_{0}^{-}$, the only new rule is $\langle(\&) \multimap\rangle$ (see [de Groote et al. 2004]). In this paper, the authors did not provide a proof of soundness/completeness of the system s-IMELL ${ }_{0}^{-}$, leaving it to the reader. Here we present a full proof of soundness/completeness for our extension G-elLL not only to please the reader but also to derive completeness of the fragment w.r.t. trivial phase semantics.

\subsection{Completeness results for elLL}

Even though validity in elLL is the same as in the whole ILL (established for instance by a proof in S-ILL), here we show that in this specific fragment, validity is also sound and complete both w.r.t. the system G-elLL and w.r.t. free monoidal trivial phase semantics.

LEMma 4.2. Every proof of a sequent in G-elLL can be transformed into a proof (of the same sequent) which uses only rules $\langle\mathrm{id}\rangle,\langle\mathrm{w}\rangle,\langle\mathrm{c}\rangle,\left\langle\multimap_{L}\right\rangle,\left\langle\multimap_{R}\right\rangle,\left\langle!_{L}\right\rangle$ and $\left\langle \&_{R}\right\rangle$ of S-ILL.

Proof. We proceed by induction on the proofs in G-elLL and by case analysis, depending on the last rule applied. Let $n$ be the cardinal of the multiset $\Sigma$. For each rule of G-elLL, we propose the corresponding (open) proof tree in S-ILL:

- case of rule $\langle\mathrm{Ax}\rangle$ :

$$
\begin{aligned}
& \frac{}{u \vdash u}\langle\mathrm{id}\rangle \\
& \frac{\mathrm{a}}{\vdots} \quad \text { applied } n \text { times } \\
& \frac{\mathrm{l} \Sigma, u \vdash u}{\mathrm{w}}\langle\mathrm{w}\rangle
\end{aligned}
$$

- case of rule $\langle\multimap\rangle$ :

$$
\begin{gathered}
\frac{! \Sigma, \Gamma \vdash u \quad \overline{v \vdash v}}{! \Sigma, \Gamma, u \multimap v \vdash v}\left\langle\multimap_{L}\right\rangle \\
\frac{! \Sigma, \Gamma, !(u \multimap v) \vdash v}{! \Sigma, \Gamma \vdash v}\left\langle!_{L}\right\rangle \\
\langle\mathrm{c}\rangle
\end{gathered}
$$

- case of rule $\langle(\multimap)-\rangle\rangle$ :

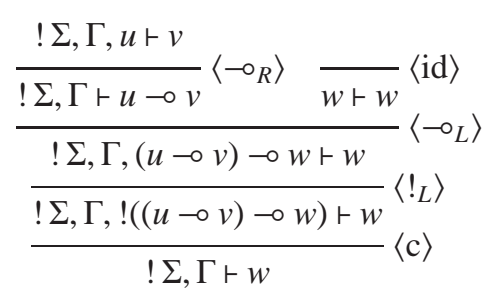


- case of rule $\langle\multimap(-\circ)\rangle$ :

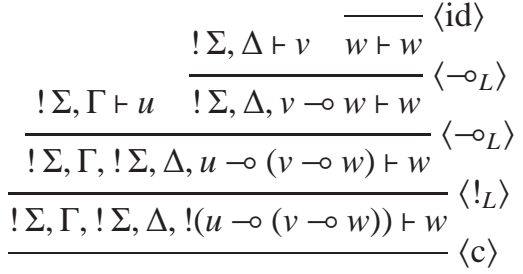

$$
\begin{aligned}
& \begin{array}{c}
\vdots \quad \text { applied } n+1 \text { times } \\
1 \Sigma \Gamma, \Delta+w
\end{array}
\end{aligned}
$$

- case of rule $\langle(\&) \multimap\rangle$ :

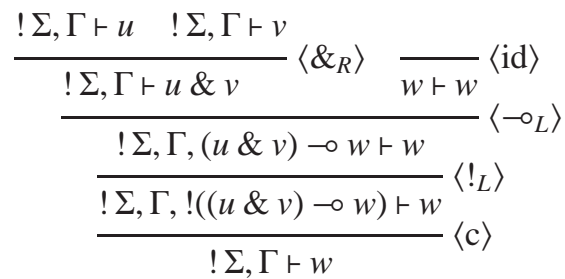

Combining those (open) proof trees, it is obvious to design a recursive algorithm which transforms G-elLL proofs into S-ILL proofs.

LEMma 4.3. If the sequent $! \Sigma, \Gamma+c$ of elLL is valid in every free monoidal trivial phase semantic interpretation then it has a proof in G-elLL.

Proof. Let us consider a fixed multiset $\Sigma=\sigma_{1}, \ldots, \sigma_{k}$ of $(-\circ, \&)$-elementary formulae. We consider the free commutative monoid over the set of logical variables $M=\mathbb{M}_{\mathrm{f}}(\mathrm{Var})$, i.e. the set of finite multisets of logical variables endowed with multiset addition (denoted by the comma) as monoidal composition and with the empty multiset (denoted $\pi=\lfloor\emptyset\rfloor$ ) as neutral element. We write $\lfloor a, a, b\rfloor$ for the multiset composed of two occurrences of $a$ and one of $b$. Let us define the free monoid $(\mathrm{M}, \star, \pi)$ of class $\mathrm{FM}$ where $\mathrm{M}=\mathbb{M}_{\mathrm{f}}(\operatorname{Var}), \pi=\lfloor\emptyset\rfloor$ and $\star: \mathrm{M} \times \mathrm{M} \longrightarrow \mathbb{P}(\mathrm{M})$ is defined by $\lfloor\Gamma\rfloor \star\lfloor\Delta\rfloor=\{\lfloor\Gamma, \Delta\rfloor\} .5$ The adjoint of $\star$ is denoted $-\star$.

We define the following semantic interpretation in the trivial phase space based on $(\mathrm{M}, \star, \pi)$ :

$$
\llbracket c \rrbracket=\{\lfloor\Gamma\rfloor \in \mathrm{M} \mid ! \Sigma, \Gamma \vdash c \text { has a proof in G-elLL }\} \quad \text { for } c \in \operatorname{Var}
$$

Let us now show that $\pi \in \llbracket \sigma_{i} \rrbracket$ holds for any $\sigma_{i} \in \Sigma$. We pick one $\sigma_{i} \in \Sigma$ and proceed by case analysis.

- if $\sigma_{i}=u \multimap v$. Then $\pi \in \llbracket u \multimap v \rrbracket$ iff $\lfloor\emptyset\rfloor \star \llbracket u \rrbracket \subseteq \llbracket v \rrbracket$ iff $\llbracket u \rrbracket \subseteq \llbracket v \rrbracket$. So let us consider one $\lfloor\Gamma\rfloor$ such that $\lfloor\Gamma\rfloor \in \llbracket u \rrbracket$ and prove that $\lfloor\Gamma\rfloor \in \llbracket v \rrbracket$. By definition of $\llbracket u \rrbracket$, the sequent $! \Sigma, \Gamma \vdash u$ has a proof in G-elLL. Then, by rule $\langle-\rangle$, the sequent $! \Sigma, \Gamma \vdash v$ has a proof in G-elLL. So we deduce $\lfloor\Gamma\rfloor \in \llbracket v \rrbracket$. Hence $\llbracket u \rrbracket \subseteq \llbracket v \rrbracket$ and we obtain $\pi \in \llbracket \sigma_{i} \rrbracket$.

- if $\sigma_{i}=(u \multimap v) \multimap w$. We have $\pi \in \llbracket(u \multimap v) \multimap w \rrbracket$ iff $\llbracket u \rrbracket-\star \llbracket v \rrbracket \subseteq \llbracket w \rrbracket$. Let use choose $\lfloor\Gamma\rfloor \in \llbracket u \rrbracket-\star \llbracket v \rrbracket$. Then $\{\lfloor\Gamma\rfloor\} \star \llbracket u \rrbracket \subseteq \llbracket v \rrbracket$. By rule $\langle\mathrm{Ax}\rangle, ! \Sigma, u \vdash u$ has a proof in G-elLL and thus $\lfloor u\rfloor \in \llbracket u \rrbracket$. Thus $\{\lfloor\Gamma, u\rfloor\}=\lfloor\Gamma\rfloor \star\lfloor u\rfloor \subseteq \llbracket v \rrbracket$. Thus ! $\Sigma, \Gamma, u \vdash v$ has a proof in G-elLL. By rule $\langle(\multimap) \multimap\rangle$, $! \Sigma, \Gamma \vdash w$ has a proof in G-elLL. We conclude $\lfloor\Gamma\rfloor \in \llbracket w \rrbracket$. Thus $\llbracket u \rrbracket-\star \llbracket v \rrbracket \subseteq \llbracket w \rrbracket$ holds, hence $\pi \in \llbracket \sigma_{i} \rrbracket$.

${ }^{5}$ Here, $\Gamma \mapsto\lfloor\Gamma\rfloor$ is the identity map on $\mathbb{M}_{\mathrm{f}}$ (Var) but the extra notation $\lfloor\cdot\rfloor$ in the expression $\{\lfloor\Gamma, \Delta\rfloor\}$ has the side effect of removing the ambiguity on the denotation of the comma: here, it denotes the composition of multisets, not the addition of elements in a set. 
- if $\sigma_{i}=u \multimap(v \multimap w)$. We have $\pi \in \llbracket u \multimap(v \multimap w) \rrbracket$ iff $\llbracket u \rrbracket \star \llbracket v \rrbracket \subseteq \llbracket w \rrbracket$. Let us choose $\lfloor\Gamma\rfloor \in \llbracket u \rrbracket$ and $\lfloor\Delta\rfloor \in \llbracket v \rrbracket$ and let us prove $\lfloor\Gamma\rfloor \star\lfloor\Delta\rfloor \subseteq \llbracket w \rrbracket$. Both $! \Sigma, \Gamma \vdash u$ and $! \Sigma, \Delta \vdash v$ have a proof in G-elLL. By rule $\langle\multimap(-)\rangle$, the sequent ! $\Sigma, \Gamma, \Delta \vdash w$ has a proof in G-elLL. Thus $\lfloor\Gamma\rfloor \star\lfloor\Delta\rfloor=\{\lfloor\Gamma, \Delta\rfloor\} \subseteq \llbracket w \rrbracket$. We deduce $\llbracket u \rrbracket \star \llbracket v \rrbracket \subseteq \llbracket w \rrbracket$ and thus conclude $\pi \in \llbracket \sigma_{i} \rrbracket$.

— if $\sigma_{i}=(u \& v) \multimap w$. We have $\pi \in \llbracket(u \& v) \multimap w \rrbracket$ iff $\llbracket u \rrbracket \cap \llbracket v \rrbracket \subseteq \llbracket w \rrbracket$. If $\lfloor\Gamma\rfloor \in \llbracket u \rrbracket \cap \llbracket v \rrbracket$ then both $! \Sigma, \Gamma \vdash u$ and $! \Sigma, \Gamma \vdash v$ have a proof in G-elLL. By rule $\langle(\&) \multimap\rangle$, the sequent $! \Sigma, \Gamma \vdash w$ has a proof in G-elLL. Thus $\lfloor\Gamma\rfloor \in \llbracket w \rrbracket$. We have proved that $\llbracket u \rrbracket \cap \llbracket v \rrbracket \subseteq \llbracket w \rrbracket$ and we conclude $\pi \in \llbracket \sigma_{i} \rrbracket$.

So, for any $i \in[1, k]$ the inclusion $\pi \in \llbracket \sigma_{i} \rrbracket$ holds and as a consequence, $\pi \in \llbracket ! \sigma_{i} \rrbracket$ because the identity $\llbracket ! \sigma_{i} \rrbracket=\{\pi\} \cap \llbracket \sigma_{i} \rrbracket$ holds in trivial phase semantics. Let us consider $\Gamma=\left\lfloor a_{1}, \ldots, a_{p}\right\rfloor$ and suppose that the sequent $! \Sigma, \Gamma \vdash c$ of elLL is valid in every free monoidal trivial phase semantics interpretation. As a particular case, it is valid in the interpretation $(M, \star, \pi, \llbracket \cdot \rrbracket)$ and thus the inclusion

$$
\llbracket ! \sigma_{1} \rrbracket \star \cdots \star \llbracket ! \sigma_{k} \rrbracket \star \llbracket a_{1} \rrbracket \star \cdots \star \llbracket a_{p} \rrbracket \subseteq \llbracket c \rrbracket
$$

holds. By rule $\langle\mathrm{Ax}\rangle$, for any $i \in[1, p]$ the sequent $! \Sigma, a_{i} \vdash a_{i}$ has a proof in G-elLL and thus the relation $\left\lfloor a_{i}\right\rfloor \in \llbracket a_{i} \rrbracket$ holds. Also remember that for any $i \in[1, k]$, we have $\lfloor\emptyset\rfloor=\pi \in \llbracket ! \sigma_{i} \rrbracket$. So

$$
\lfloor\Gamma\rfloor \in\left\{\left\lfloor a_{1}, \ldots, a_{p}\right\rfloor\right\}=\lfloor\emptyset\rfloor \star \cdots \star\lfloor\emptyset\rfloor \star\left\lfloor a_{1}\right\rfloor \star \cdots \star\left\lfloor a_{p}\right\rfloor \subseteq \llbracket c \rrbracket
$$

holds and we conclude that $! \Sigma, \Gamma \vdash c$ has a proof in G-elLL.

THEOREM 4.4. The system G-elLL is sound and complete for the fragment elLL. Given a class $\mathrm{X} \in\{\mathrm{ND}, \mathrm{PD}, \mathrm{TD}, \mathrm{FM}\}$, the trivial phase semantics over the class $\mathrm{X}$ is sound and complete for the fragment elLL.

Proof. Consider the following inclusion sequence

$$
\mathrm{elLL}_{g} \subseteq \mathrm{elLL}_{p} \subseteq \mathrm{elLL}_{\mathrm{ND}}^{t} \subseteq \mathrm{elLL}_{\mathrm{PD}}^{t} \subseteq \mathrm{elLL}_{\mathrm{TD}}^{t} \subseteq \mathrm{elLL}_{\mathrm{FM}}^{t} \subseteq \mathrm{elLL}_{g}
$$

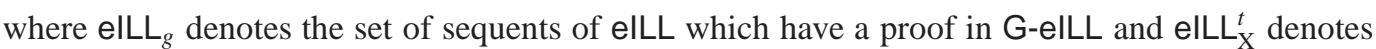
the set of sequents which are valid in every trivial phase semantic interpretation of the class X. The inclusion elLL $g \subseteq$ elLL ${ }_{p}$ is a direct consequence of Lemma 4.2. The inclusion elLL el $_{p}$ elLL $_{\text {ND }}^{t}$ is a particular case of Theorem 3.3. The inclusion sequence elLL ${ }_{\mathrm{ND}}^{t} \subseteq \mathrm{elLL}_{\mathrm{PD}}^{t} \subseteq \mathrm{elLL}_{\mathrm{TD}}^{t} \subseteq \mathrm{elLL}_{\mathrm{FM}}^{t}$ is an obvious consequence of the inclusions FM $\subseteq$ TD $\subseteq$ PD $\subseteq$ ND between classes of non-deterministic monoids. The last inclusion elLL $t_{\mathrm{FM}}^{t} \subseteq \mathrm{elLL}_{g}$ is the result of Lemma 4.3.

Remark: the problem of the completeness of the fragment elLL w.r.t. trivial heap semantics requires bisimulating free monoids with heap monoids and will be addressed in Section 7.2.

\section{THE UNDECIDABILITY OF ELEMENTARY INTUITIONISTIC LINEAR LOGIC}

We propose an encoding of two counter Minsky machines in the fragment elLL of ILL. The first encoding of Minsky machines in Linear Logic was done by Kanovich in the $(!, \oplus)$-Horn fragment of ILL [Kanovich 1994; 1995]. In this encoding, the recovery of computations from proofs is obtained through some form of proof normalization and the $\oplus$ additive connective is used to simulate forking. Lafont later showed that the use of proof normalization can be avoided and replaced by a phase semantics argument [Lafont 1996; Lafont and Scedrov 1996]. In our encoding of Minsky machines in elLL, the \& connective is used to simulate forking and we will show that a trivial phase semantics argument is sufficient to recover computability from provability.

\subsection{Two counter Minsky machines}

Let $\mathrm{a}$ and $\mathrm{b}$ be two distinct counter symbols. A (deterministic) two counter Minsky machine is a pair $\mathfrak{M}=(l, \psi)$ where $l>0$ is a strictly positive natural number of instructions and

$$
\psi:[1, l] \longrightarrow\{+\} \times\{\mathrm{a}, \mathrm{b}\} \times[0, l] \mid\{-\} \times\{\mathrm{a}, \mathrm{b}\} \times[0, l] \times[0, l]
$$


is a total map representing the list of instructions. Here, | represents the (disjoint) set sum. Minsky machines instructions (incrementation, zero test/decrementation) are encoded as illustrated in the two following examples:

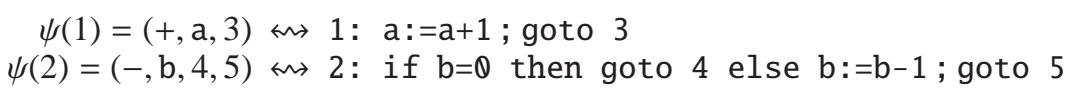

Given a two counter Minsky machine $\mathfrak{M}=(l, \psi)$, we define the set $\mathrm{S}(\mathfrak{M})$ of states of the machine by $S(\mathfrak{M})=[0, l] \times \mathbb{N} \times \mathbb{N}$ which collects the current instruction and the values of the counters a and b. With the following notations:

$$
\overline{\mathrm{a}}=(1,0) \quad \overline{\mathrm{b}}=(0,1) \quad(m, n)_{\mathrm{a}}=m \quad(m, n)_{\mathrm{b}}=n
$$

we define a (binary) transition relation between states $\rightarrow_{\mathfrak{M}} \subseteq \mathrm{S}(\mathfrak{M}) \times \mathrm{S}(\mathfrak{M})$. For any two states $(i, m, n)$ and $\left(i^{\prime}, m^{\prime}, n^{\prime}\right)$, the relation $(i, m, n) \rightarrow_{\mathfrak{M}}\left(i^{\prime}, m^{\prime}, n^{\prime}\right)$ holds if

$$
\begin{aligned}
\psi(i) & =\left(+, x, i^{\prime}\right) \text { and }\left(m^{\prime}, n^{\prime}\right)=(m, n)+\bar{x} \\
\text { or } \psi(i) & =\left(-, x, i^{\prime}, k\right),(m, n)_{x}=0 \text { and }\left(m^{\prime}, n^{\prime}\right)=(m, n) \\
\text { or } \psi(i) & =\left(-, x, j, i^{\prime}\right),(m, n)_{x} \neq 0 \text { and }\left(m^{\prime}, n^{\prime}\right)+\bar{x}=(m, n)
\end{aligned}
$$

holds for some $x \in\{\mathrm{a}, \mathrm{b}\}$ and some $j, k \in[0, l]$. Notice that $(i, m, n) \rightarrow_{\mathfrak{M}}\left(i^{\prime}, m^{\prime}, n^{\prime}\right)$ does not hold if $i=0$ because $\psi(0)$ is not defined. Let $\rightarrow_{\mathfrak{M}}^{\star}$ be the reflexive and transitive closure of the relation $\rightarrow_{\mathfrak{M}}$. We say that the machine $\mathfrak{M}$ accepts the input $(m, n)$ if starting from the state $(1, m, n)$, there exists a sequence of transitions leading to the state $(0,0,0)$ and we define the set $A(\mathfrak{M})$ of accepted inputs:

$$
\mathrm{A}(\mathfrak{M})=\left\{(m, n) \in \mathbb{N} \times \mathbb{N} \mid(1, m, n) \rightarrow_{\mathfrak{M}}^{\star}(0,0,0)\right\}
$$

Theorem 5.1 (Minsky). There exists a two counter Minsky machine $\mathfrak{M}$ for which the set $\mathrm{A}(\mathfrak{M})$ of accepted inputs is not recursive [Minsky 1961].

\subsection{The encoding of two counter Minsky machines}

Let us consider the two counter symbols a and $\mathrm{b}$ as two (different) logical variables and let us choose two new variables $\underline{a}$ and $\underline{b}$ so that the set $\{a, b, \underline{a}, \underline{b}\} \subseteq$ Var has cardinal four. Let us choose an infinite set $^{6}$ of new logical variables $\left\{\mathrm{q}_{i} \mid i \in \mathbb{N}\right\}$ such that $\mathrm{q}_{i} \neq \mathrm{q}_{j}$ unless $i=j$ and $\{\mathrm{a}, \mathrm{b}, \underline{\mathrm{a}}, \underline{\mathrm{b}}\} \cap\left\{\mathrm{q}_{i} \mid i \in \mathbb{N}\right\}=$ $\emptyset$. Let $\Sigma_{0}$ be the following multiset composed of five $(-, \&)$-elementary formulae:

$$
\Sigma_{0}=\left\{\mathrm{a} \multimap(\underline{\mathrm{a}} \multimap \underline{\mathrm{a}}), \mathrm{b} \multimap(\underline{\mathrm{b}} \multimap \underline{\mathrm{b}}),(\mathrm{a} \multimap \mathrm{a}) \multimap \underline{\mathrm{a}},(\mathrm{a} \multimap \mathrm{a}) \multimap \underline{\mathrm{b}},(\mathrm{a} \multimap \mathrm{a}) \multimap \mathrm{q}_{0}\right\}
$$

Given a Minsky machine $\mathfrak{M}=(l, \psi)$, for $i \in[1, l]$, we define the multisets $\Sigma_{1}, \ldots, \Sigma_{l}$ of $(-, \&)$ elementary formulae by:

$$
\begin{aligned}
& \Sigma_{i}=\left\{\left(x \multimap \mathrm{q}_{j}\right) \multimap \mathrm{q}_{i}\right\} \quad \text { when } \psi(i)=(+, x, j) \\
& \text { and } \Sigma_{i}=\left\{\left(\underline{x} \& \mathrm{q}_{j}\right) \multimap \mathrm{q}_{i}, x \multimap\left(\mathrm{q}_{k} \multimap \mathrm{q}_{i}\right)\right\} \text { when } \psi(i)=(-, x, j, k)
\end{aligned}
$$

Let $\Sigma_{\mathfrak{M}}$ be the multiset $\Sigma_{\mathfrak{M}}=\Sigma_{0}, \Sigma_{1}, \ldots, \Sigma_{l}$. Given a natural number $n$ and a logical variable $x \in$ $\{\mathrm{a}, \mathrm{b}\}$, we define $x^{n}=x, x, \ldots, x$ as the multiset composed of $n$ occurrences of the variable $x$. Then, it is trivial to verify that for any natural numbers $m, n$ and any $i \in[0, l]$, the sequent $! \Sigma_{\mathfrak{M}}, \mathrm{a}^{m}, \mathrm{~b}^{n} \vdash \mathrm{q}_{i}$ belongs to the fragment elLL.

Let us now consider a fixed Minsky machine $\mathfrak{M}=(l, \psi)$. Then we denote $\Sigma_{\mathfrak{M}}\left(\right.$ resp. $\left.\rightarrow_{\mathfrak{M}}\right)$ simply by $\Sigma$ (resp. $\rightarrow$ ). We prove four main intermediate results.

Proposition 5.2. For any $m, n \in \mathbb{N}$, the sequents $! \Sigma, \mathrm{a}^{m}+\underline{\mathrm{a}}$ and $! \Sigma, \mathrm{b}^{n}+\underline{\mathrm{b}}$ are provable in $\mathrm{G}-\mathrm{elLL}$.

\footnotetext{
${ }^{6}$ For our particular purpose, we only need as many $\mathrm{q}_{i}$ 's as there are instructions in the Minsky machine obtained from Theorem 5.1.
} 
Proof. Here is a suitable proof tree for the case with a/a.

$$
\begin{aligned}
& \begin{array}{ll}
\frac{\overline{! \Sigma, a}, \mathrm{a} \vdash \mathrm{a}}{\operatorname{l}, \mathrm{a} \vdash \mathrm{a}}\langle\mathrm{Ax}\rangle & \frac{\mathrm{a} \rightarrow \mathrm{a}) \multimap \underline{\mathrm{a}} \in \Sigma}{! \Sigma \vdash \underline{\mathrm{a}}} \mathrm{a} \multimap(\underline{\mathrm{a}} \multimap \underline{\mathrm{a}}) \in \Sigma
\end{array} \\
& \text { : applied } m-1 \text { times }
\end{aligned}
$$

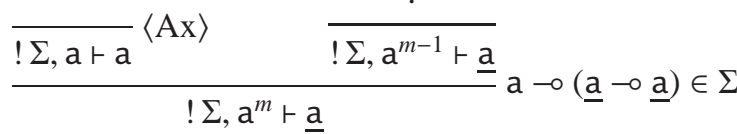

The case of $b / \underline{b}$ is similar. Here is a suitable proof tree:

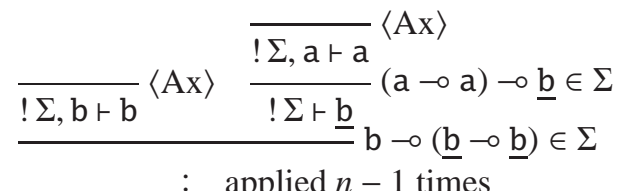



In fact, these are the only possible proof trees but the demonstration of this uniqueness result is left to the reader.

Lemma 5.3. For any $r, m, n \in \mathbb{N}$ and any $i \in[0, l]$, if $(i, m, n) \rightarrow^{r}(0,0,0)$ then the sequent ! $\Sigma, \mathrm{a}^{m}, \mathrm{~b}^{n}+\mathrm{q}_{i}$ is provable in $\mathrm{G}-\mathrm{elLL}$.

Proof. We proceed by induction on the length $r$ of the transition sequence $(i, m, n) \rightarrow^{r}(0,0,0)$ leading to the accepting state.

If $r=0$ then we have $(i, m, n)=(0,0,0)$. The sequent $! \Sigma \vdash \mathrm{q}_{0}$ has the following proof tree:

$$
\frac{\overline{! \Sigma, \mathrm{a} \vdash \mathrm{a}}\langle A \mathrm{x}\rangle}{! \Sigma \vdash \mathrm{q}_{0}}(\mathrm{a} \multimap \mathrm{a}) \multimap \mathrm{q}_{0} \in \Sigma
$$

Let us now consider a transition sequence $(i, m, n) \rightarrow\left(i^{\prime}, m^{\prime}, n^{\prime}\right) \rightarrow^{r}(0,0,0)$ of length $r+1$. By induction hypothesis, let $P$ be a proof tree for the sequent $! \Sigma, \mathrm{a}^{m^{\prime}}, \mathrm{b}^{n^{\prime}}+\mathrm{q}_{i^{\prime}}$. We consider the $3 \times 2$ possible cases for $(i, m, n) \rightarrow\left(i^{\prime}, m^{\prime}, n^{\prime}\right)$.

- if $\psi(i)=\left(+, \mathrm{a}, i^{\prime}\right)$ and $\left(m^{\prime}, n^{\prime}\right)=(m, n)+\overline{\mathrm{a}}$. Then $m^{\prime}=m+1$ and $n^{\prime}=n$. We provide the following proof tree for $! \Sigma, \mathrm{a}^{m}, \mathrm{~b}^{n}+\mathrm{q}_{i}$ :

$$
\frac{P}{! \Sigma, \mathrm{a}^{m}, \mathrm{~b}^{n}, \mathrm{a} \vdash \mathrm{q}_{i^{\prime}}}\left(\mathrm{a} \multimap \mathrm{q}_{i^{\prime}}\right) \multimap \mathrm{q}_{i} \in \Sigma
$$

— if $\psi(i)=\left(+, \mathrm{b}, i^{\prime}\right), m^{\prime}=m$ and $n^{\prime}=n+1$. Here is a proof tree for $! \Sigma, \mathrm{a}^{m}, \mathrm{~b}^{n} \vdash \mathrm{q}_{i}$ :

$$
\frac{P}{! \Sigma, \mathrm{a}^{m}, \mathrm{~b}^{n}, \mathrm{~b} \vdash \mathrm{q}_{i^{\prime}}}\left(\mathrm{b} \multimap \mathrm{q}_{i^{\prime}}\right) \multimap \mathrm{q}_{i} \in \Sigma
$$

- if $\psi(i)=\left(-, \mathrm{a}, i^{\prime}, k\right),(m, n)_{\mathrm{a}}=0$ and $\left(m^{\prime}, n^{\prime}\right)=(m, n)$. Then $m=m^{\prime}=0$ and $n=n^{\prime}$. Let $Q$ be a proof tree for $! \Sigma, \mathrm{b}^{n} \vdash \underline{\mathrm{b}}$ according to Proposition 5.2. We provide the following proof tree for 
$! \Sigma, \mathrm{b}^{n}+\mathrm{q}_{i}:$

$$
\frac{\frac{Q}{! \Sigma, \mathrm{b}^{n}+\underline{\mathrm{b}}} \frac{P}{! \Sigma, \mathrm{b}^{n}+\mathrm{q}_{i^{\prime}}}\left(\underline{\mathrm{b}} \& \mathrm{q}_{i^{\prime}}\right) \multimap \mathrm{q}_{i} \in \Sigma}{! \Sigma, \mathrm{b}^{n}+\mathrm{q}_{i}}
$$

— if $\psi(i)=\left(-, \mathrm{b}, i^{\prime}, k\right), m=m^{\prime}$ and $n=n^{\prime}=0$. Let $Q$ be a proof tree for $! \Sigma, \mathrm{a}^{m} \vdash \underline{\mathrm{a}}$ according to Proposition 5.2. Here is a proof tree for $! \Sigma, \mathrm{b}^{n}+\mathrm{q}_{i}$ :

$$
\frac{\frac{Q}{! \Sigma, \mathrm{a}^{m}+\underline{\mathrm{a}}} \frac{P}{! \Sigma, \mathrm{a}^{m}+\mathrm{q}_{i^{\prime}}}\left(\underline{\mathrm{a}} \& \mathrm{q}_{i^{\prime}}\right) \multimap \mathrm{q}_{i} \in \Sigma}{! \Sigma, \mathrm{a}^{m}+\mathrm{q}_{i}}
$$

— if $\psi(i)=\left(-, \mathrm{a}, j, i^{\prime}\right),(m, n)_{\mathrm{a}} \neq 0$ and $\left(m^{\prime}, n^{\prime}\right)+\overline{\mathrm{a}}=(m, n)$. Then $m=m^{\prime}+1$ and $n=n^{\prime}$. We provide the following proof tree for $! \Sigma, \mathrm{a}, \mathrm{a}^{m^{\prime}}, \mathrm{b}^{n^{\prime}}+\mathrm{q}_{i}$ :

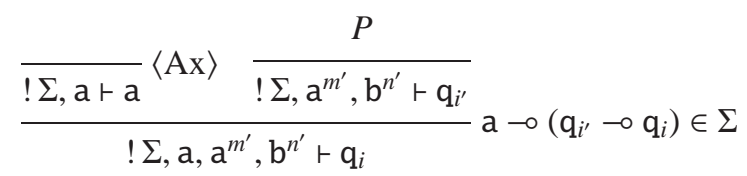

— if $\psi(i)=\left(-, \mathrm{b}, j, i^{\prime}\right), m^{\prime}=m$ and $n^{\prime}+1=n$. Here is a proof tree for $! \Sigma, \mathrm{a}^{m^{\prime}}, \mathrm{b}, \mathrm{b}^{n^{\prime}} \vdash \mathrm{q}_{i}$ :

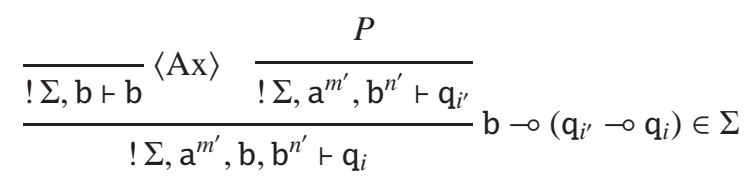

In any case we obtain a proof tree for $! \Sigma, \mathrm{a}^{m}, \mathrm{~b}^{n} \vdash \mathrm{q}_{i}$ which fulfills the induction step. Again, but this is left to the reader, it can be demonstrated that the proof tree recursively build from the transition sequence $(i, m, n) \rightarrow^{r}(0,0,0)$ is the unique proof tree for the sequent $! \Sigma, \mathrm{a}^{m}, \mathrm{~b}^{n} \vdash \mathrm{q}_{i}$.

Let us now consider the following trivial phase semantics interpretation. Consider the product monoid $(\mathbb{N} \times \mathbb{N},+,(0,0))$. We define $x \circ y=\{x+y\}$ and thus $(\mathbb{N} \times \mathbb{N}, \circ,(0,0))$ is a total deterministic monoid. Every subset of $\mathbb{N} \times \mathbb{N}$ is closed in trivial phase semantics and we define

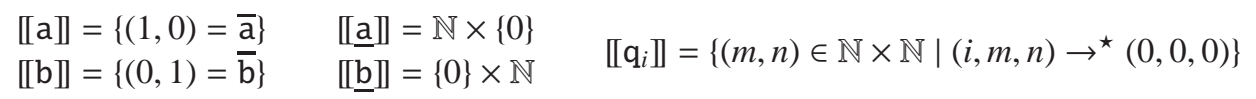

It is crucial that variables $\mathrm{a}, \mathrm{b}, \underline{\mathrm{a}}, \underline{\mathrm{b}}, \mathrm{q}_{0}, \mathrm{q}_{1}, \ldots, \mathrm{q}_{l}$ were chosen distinct from one another for this definition to be valid. Let us now consider the trivial phase semantics interpretation of the compound formulae of $\Sigma$.

Proposition 5.4. For any $\sigma \in \Sigma$, $\llbracket ! \sigma \rrbracket=\{(0,0)\}$ holds.

Proof. As the identity $\llbracket ! \sigma \rrbracket=\{(0,0)\} \cap \llbracket \sigma \rrbracket$ holds in trivial phase semantics, it is necessary and sufficient to prove that $(0,0) \in \llbracket \sigma \rrbracket$ holds for any $\sigma \in \Sigma$.

First let us prove that $\llbracket a \multimap a \rrbracket=\{(0,0)\}$. Indeed, $(m, n) \in \llbracket a \multimap a \rrbracket$ iff $(m, n) \circ \llbracket a \rrbracket \subseteq \llbracket a \rrbracket$ iff $(m, n) \circ\{(1,0)\} \subseteq\{(1,0)\}$ iff $\{(m+1, n)\} \subseteq\{(1,0)\}$ iff $(m, n)=(0,0)$. Then $\llbracket(\mathrm{a} \multimap \mathrm{a}) \multimap x \rrbracket=$ $\{(0,0)\} \multimap \llbracket x \rrbracket=\llbracket x \rrbracket$ for any variable $x$, in particular for $x \in\left\{\underline{\mathrm{a}}, \underline{\mathrm{b}}, \mathrm{q}_{0}\right\}$. Also $(m, n) \in \llbracket \mathrm{a} \multimap(\underline{\mathrm{a}}-\underline{\mathrm{a}}) \rrbracket$ iff $(m, n) \circ\{(1,0)\} \circ \mathbb{N} \times\{0\} \subseteq \mathbb{N} \times\{0\}$ iff $n=0$. Thus $\llbracket a \multimap(\underline{a}-\underline{a}) \rrbracket=\mathbb{N} \times\{0\}$. By a similar argument, we get $\llbracket \mathrm{b} \multimap(\mathrm{b} \multimap \underline{\mathrm{b}}) \rrbracket=\{0\} \times \mathbb{N}$. So for any formula $\sigma \in \bar{E} \Sigma_{0}$, we have $(0,0) \in \llbracket \sigma \rrbracket$.

Let us consider the formulae in $\Sigma_{i}$ for $i \in[1, l]$. Let us prove that the relation $(0,0) \in \llbracket \sigma \rrbracket$ holds for any $\sigma \in \Sigma_{i}$ by case analysis: 
- if $\psi(i)=(+, x, j)$. Let us show $(0,0) \in \llbracket\left(x \multimap \mathrm{q}_{j}\right) \multimap \mathrm{q}_{i} \rrbracket$, i.e. $\llbracket x \multimap \mathrm{q}_{j} \rrbracket \subseteq \llbracket \mathrm{q}_{i} \rrbracket$. Let us consider $(m, n) \in \llbracket x \multimap \mathrm{q}_{j} \rrbracket$. Then $\{(m, n)+\bar{x}\}=\{(m, n)\} \circ \llbracket x \rrbracket \subseteq \llbracket \mathrm{q}_{j} \rrbracket$ and thus $\left(m^{\prime}, n^{\prime}\right)=(m, n)+\bar{x} \in \llbracket \mathrm{q}_{j} \rrbracket$. Thus we have $(i, m, n) \rightarrow\left(j, m^{\prime}, n^{\prime}\right) \rightarrow^{\star}(0,0,0)$. We conclude $(m, n) \in \llbracket \mathrm{q}_{i} \rrbracket$.

- if $\psi(i)=(-, x, j, k)$. Let us first show that $(0,0) \in \llbracket\left(x \& \mathrm{q}_{j}\right) \multimap \mathrm{q}_{i} \rrbracket$, i.e. $\llbracket x \rrbracket \cap \llbracket \mathrm{q}_{j} \rrbracket \subseteq \llbracket \mathrm{q}_{i} \rrbracket$. Let us consider $(m, n) \in \llbracket \underline{x} \rrbracket \cap \llbracket \mathrm{q}_{j} \rrbracket$. Then $(m, n)_{x}=0$ and $(j, m, n) \rightarrow^{\star}(0,0,0)$. Thus $(i, m, n) \rightarrow$ $(j, m, n) \rightarrow^{\star}(0,0,0)$ and $(m, n) \in \llbracket \mathrm{q}_{i} \rrbracket$. Hence $\llbracket \underline{x} \rrbracket \cap \llbracket \mathrm{q}_{j} \rrbracket \subseteq \llbracket \mathrm{q}_{i} \rrbracket$ holds. Let us finally show that $(0,0) \in \llbracket x \multimap\left(\mathrm{q}_{k} \multimap \mathrm{q}_{i}\right) \rrbracket$, i.e. $\llbracket x \rrbracket \circ \llbracket \mathrm{q}_{k} \rrbracket \subseteq \llbracket \mathrm{q}_{i} \rrbracket$. As $\llbracket x \rrbracket=\{\bar{x}\}$ for $x \in\{\mathrm{a}, \mathrm{b}\}$, let us choose an arbitrary pair $\left(m^{\prime}, n^{\prime}\right) \in \llbracket \mathrm{q}_{k} \rrbracket$ and define $(m, n)=\bar{x}+\left(m^{\prime}, n^{\prime}\right)$. Then $(m, n)_{x}=1+\left(m^{\prime}, n^{\prime}\right)_{x} \neq 0$ and $(i, m, n) \rightarrow\left(k, m^{\prime}, n^{\prime}\right) \rightarrow^{\star}(0,0,0)$. We obtain $(m, n) \in \llbracket \mathrm{q}_{i} \rrbracket$ and thus conclude $\bar{x}+\left(m^{\prime}, n^{\prime}\right) \in \llbracket \mathrm{q}_{i} \rrbracket$. Hence, for any $\left(m^{\prime}, n^{\prime}\right) \in \llbracket \mathrm{q}_{k} \rrbracket$ we get $\llbracket x \rrbracket \circ\left(m^{\prime}, n^{\prime}\right) \subseteq \llbracket \mathrm{q}_{i} \rrbracket$. Thus $\llbracket x \rrbracket \circ \llbracket \mathrm{q}_{k} \rrbracket \subseteq \llbracket \mathrm{q}_{i} \rrbracket$ holds

As a consequence, for any $\sigma \in \Sigma$, we obtain $(0,0) \in \llbracket \sigma \rrbracket$. The identity $\llbracket ! \sigma \rrbracket=\{(0,0)\}$ holds for any $\sigma \in \Sigma$.

Lemma 5.5. For any $m, n \in \mathbb{N}$, if $! \Sigma, \mathrm{a}^{m}, \mathrm{~b}^{n} \vdash \mathrm{q}_{1}$ is provable in $\mathrm{G}$-elLL then $(m, n) \in \mathrm{A}(\mathfrak{M})$ holds.

Proof. Let $\Sigma=\left\{\sigma_{1}, \ldots, \sigma_{r}\right\}$. We suppose that the sequent $! \Sigma, \mathrm{a}^{m}, \mathrm{~b}^{n} \vdash \mathrm{q}_{1}$ has a proof in G-elLL. By the soundness part of Theorem 4.4, in our particular total deterministic trivial phase semantics interpretation, we have

$$
\llbracket ! \sigma_{1} \rrbracket \circ \cdots \circ \llbracket ! \sigma_{r} \rrbracket \circ \llbracket \mathrm{a} \rrbracket \circ \cdots \circ \llbracket \mathrm{a} \rrbracket \circ \llbracket \mathrm{b} \rrbracket \circ \cdots \circ \llbracket \mathrm{b} \rrbracket \subseteq \llbracket \mathrm{q}_{1} \rrbracket
$$

where a occurs $m$ times and b occurs $n$ times. By Proposition 5.4 , we deduce $(m, n)=r \cdot(0,0)+$ $m .(1,0)+n \cdot(0,1) \in \llbracket \mathrm{q}_{1} \rrbracket$ and thus $(1, m, n) \rightarrow^{\star}(0,0,0)$ holds.

From Lemma 5.3 and Lemma 5.5, we obtain as a direct consequence the following theorem which characterizes Minsky machine acceptance in terms of provability in G-elLL.

THEOREM 5.6. For any two counter Minsky machine $\mathfrak{M}$ and for any pair $m, n \in \mathbb{N}$, we have $(m, n) \in \mathrm{A}(\mathfrak{M})$ if and only if the sequent $! \Sigma_{\mathfrak{M}}, \mathrm{a}^{m}, \mathrm{~b}^{n}+\mathrm{q}_{1}$ is provable in $\mathrm{G}$-elLL.

We point out that the form (\&) - 0 is used here to encode forking in a way similar Kanovich does with $\oplus$ (see [Kanovich 1995]). The reader may have noticed that more than the simple encoding of computability with provability, we can even show that computations and proofs match one to one. Even though this result is not necessary to our argumentation, this suggests that the system G-elLL is a natural choice to illustrate the relations between Minsky machines and linear logic, and may be more straightforward than the (!, $\oplus)$-Horn fragment [Kanovich 1995].

\subsection{The undecidability of elLL}

Whereas the decidability of s-IMELL ${ }_{0}^{-0}$ is still unclear (but nevertheless known to be equivalent to the decidability of MELL [de Groote et al. 2004]), we have proved that the simple addition of the form (\&) $\multimap$ to $\mathrm{s}-\mathrm{IMELL}-0$ is sufficient to encode forking and thus, computations of Minsky machines.

THEOREM 5.7. Validity is undecidable in the elementary fragment of ILL.

Proof. By Theorem 5.1, let $\mathfrak{M}$ be a two counter Minsky machine such that $A(\mathfrak{M})$ is not recursive. Compute $\Sigma_{\mathfrak{M}}$. If there is an algorithm that discriminates between provable and unprovable sequents of elLL, use it to decide

$$
\mathrm{A}(\mathfrak{M})=\left\{(m, n) \in \mathbb{N} \times \mathbb{N} \mid ! \Sigma, \mathrm{a}^{m}, \mathrm{~b}^{n}+\mathrm{q}_{1} \text { is provable in G-elLL }\right\}
$$

This identity is a direct consequence of Theorem 5.6. Thus $A(\mathfrak{M})$ would be recursive. We obtain a contradiction.

We point out that the model through which the faithfulness of the encoding is obtained (see Lemma 5.5) is based on the free monoid $\mathbb{N} \times \mathbb{N}$. So let us denote by elLL $_{\mathbb{N} \times \mathbb{N}}^{t}$ the set of sequents which are valid in every trivial phase semantic interpretation over the free monoid $(\mathbb{N} \times \mathbb{N},+,(0,0))$. We obtain the following stronger theorem: 
Theorem 5.8. Let $\mathrm{X}$ be a set of sequents in-between elLL $_{p}$ and elLL $_{\mathbb{N} \times \mathbb{N}}^{t}$, i.e. which satisfies elLL $_{p} \subseteq \mathrm{X} \subseteq$ elLL $_{\mathbb{N} \times \mathbb{N}}^{t}$. Then the set $\mathrm{X}$ is not a recursive set of sequents.

Proof. It is sufficent to prove the following equivalence:

$$
(m, n) \in \mathrm{A}(\mathfrak{M}) \quad \text { iff } \quad ! \Sigma, \mathrm{a}^{m}, \mathrm{~b}^{n}+\mathrm{q}_{1} \text { belongs to } \mathrm{X}
$$

For the if part, if $! \Sigma, \mathrm{a}^{m}, \mathrm{~b}^{n}+\mathrm{q}_{1}$ belongs to $\mathrm{X}$ then, it belongs to elLL $\mathrm{L}_{\mathbb{N} \times \mathbb{N}}^{t}$ and hence, using the same proof as in Lemma 5.5, we deduce that $(m, n) \in \mathrm{A}(\mathfrak{M})$. For the only if part, if $(m, n) \in \mathrm{A}(\mathfrak{M})$, then by Lemma 5.3 , we obtain that $! \Sigma, \mathrm{a}^{m}, \mathrm{~b}^{n} \vdash \mathrm{q}_{1}$ is provable in G-elLL. Thus, by definition, it belongs to elLL ${ }_{p}$, and as a consequence, the sequent $! \Sigma, \mathrm{a}^{m}, \mathrm{~b}^{n} \vdash \mathrm{q}_{1}$ belongs to $\mathrm{X}$.

Remark: we leave the question of the strictness of the inclusion elLL $\subseteq_{p} \operatorname{elLL}_{\mathbb{N} \times \mathbb{N}}^{t}$ as a remaining open problem.

\section{THE SEMANTICS OF BOOLEAN BI}

Boolean $\mathrm{BI}$ (denoted BBI) is the variant of intuitionistic BI [O'Hearn and Pym 1999] where the additive connectives are interpreted as Boolean connectives, contrary to (intuitionistic) BI where the additive connectives are interpreted as in propositional intuitionistic logic. The linear connectives are both interpreted as those of multiplicative intuitionistic linear logic, i.e. the multiplicative fragment of ILL. When the connectives of BBI are given a Kripke semantics (see Section 6.1) and the model belongs to the class of heap monoids HM, then we recover the core logic behind Separation Logic [Ishtiaq and O'Hearn 2001].

The syntax of $\mathrm{BBI}$ is exactly the syntax of $\mathrm{BI}$ augmented with negation, although negation could be defined by $\neg A=A \rightarrow \perp$ like in classical logic. Thus, the formulae of BBI are defined as follows. Starting from a set Var, they are freely built using the logical variables in Var, the logical constants in $\{l, T, \perp\}$, the unary connective $\neg$ or the binary connectives in $\{*, *, \wedge, \vee, \rightarrow\}$. Formally, the set of formulae is described by the following grammar:

$$
A::=v|c| \neg A \mid A \text { 米 } A \quad \text { with } v \in \operatorname{Var}, c \in\{l, \top, \perp\} \text { and } \circledast \in\{*, *, \wedge, \vee, \rightarrow\}
$$

Validity in BBI has not always been unequivocally defined. Indeed, the initial proposition of Pym [Pym 2002] was simply to add a double negation principle to the cut-free bunched proof system of BI. But of course, this does not lead to a proof-theoretically well behaved proof-system for BBI: it does not enjoy cut-elimination, sub-formula property, etc. Then, the syntax of BBI has been used as a foundation for numerous variants of Separation Logic with the common property that the additive operator $\rightarrow$ is interpreted classically whereas it is interpreted intuitionistically in BI [Ishtiaq and O'Hearn 2001; Calcagno et al. 2005]. The removal of the pre-order in the Kripke semantics is moreover necessary for the interpretation of classical negation $\neg$.

\subsection{Kripke Semantics for BBI}

In this paper, we choose to present $\mathrm{BBI}$ as a family of logics defined by their Kripke semantics rather than proof-systems. Given a non-deterministic monoid $(M, \circ, \epsilon)$ and an interpretation of propositional variables $\delta:$ Var $\longrightarrow \mathbb{P}(\mathrm{M})$, we define the Kripke forcing relation $\Vdash_{\delta}$ by induction on the structure of formulae:

$$
\begin{gathered}
m \Vdash_{\delta} \perp \text { iff never } \\
m \Vdash_{\delta} \top \text { iff always } \\
m \Vdash_{\delta} \neg A \text { iff } m \nVdash_{\delta} A \\
m \Vdash_{\delta} I \text { iff } m=\epsilon \\
m \Vdash_{\delta} v \text { iff } m \in \delta(v)
\end{gathered}
$$

$$
\begin{aligned}
& m \Vdash_{\delta} A \vee B \text { iff } m \Vdash_{\delta} A \text { or } m \Vdash_{\delta} B \\
& m \Vdash_{\delta} A \wedge B \text { iff } m \Vdash_{\delta} A \text { and } m \Vdash_{\delta} B \\
& m \Vdash_{\delta} A \rightarrow B \text { iff } m \nVdash_{\delta} A \text { or } m \Vdash_{\delta} B \\
& m \Vdash_{\delta} A * B \text { iff } \exists a, b, m \in a \circ b \text { and } a \Vdash_{\delta} A \text { and } b \Vdash_{\delta} B \\
& m \Vdash_{\delta} A \rightarrow B \text { iff } \forall a, b\left(b \in a \circ m \text { and } a \Vdash_{\delta} A\right) \Rightarrow b \Vdash_{\delta} B
\end{aligned}
$$

Definition 6.1. A formula $F$ is valid in a non-deterministic monoid $(M, \circ, \epsilon)$ if for any interpretation $\delta: \operatorname{Var} \longrightarrow \mathbb{P}(M)$ of propositional variables, the relation $m \Vdash_{\delta} F$ holds for any $m \in M$. A counter-model of the formula $F$ is given by a non-deterministic monoid $(M, \circ, \epsilon)$, an interpretation $\delta: \operatorname{Var} \longrightarrow \mathbb{P}(M)$ and an element $m \in M$ such that $m \nVdash_{\delta} F$. 
When the interpretation of variables is obvious from the context, we may simply omit the $\delta$ subscript and write $\Vdash$ instead of $\Vdash_{\delta}$. In some papers, you might find BBI defined by non-deterministic monoidal Kripke semantics [Brotherston 2010; Galmiche and Larchey-Wendling 2006], in other papers it is defined by partial but deterministic monoidal Kripke semantics and generally Separation Logic models are particular instances of partial (deterministic) monoids. See [Larchey-Wendling and Galmiche 2009] for a general discussion about these issues.

Definition 6.2. We denote by $\mathrm{BBI}_{\mathrm{X}}$ the set of formulae of $\mathrm{BBI}$ which are valid in every monoid of the class $X$. The class $X$ ranges over $\{\mathrm{ND}, \mathrm{PD}, \mathrm{TD}, \mathrm{HM}, \mathrm{FM}\}$.

On the proof-theoretic side, we briefly recall that $\mathrm{BBI}_{\mathrm{ND}}$ has been proved sound and complete w.r.t. a Hilbert proof-system [Galmiche and Larchey-Wendling 2006] and also, more recently w.r.t. a Display Logic based proof-system [Brotherston 2010] enjoying cut-elimination. $B B I_{P D}$ can be proved sound and complete w.r.t. the semantic constraints based tableaux proof-system presented in [Larchey-Wendling and Galmiche 2009] (although only the soundness proof is presented in that particular paper) and the adaptation of this tableaux system to $\mathrm{BBI}_{\mathrm{TD}}$ should be straightforward (contrary to $\mathrm{BBI}_{\mathrm{ND}}$ ).

As it turns out, the three different classes of models ND, PD and TD define three different logics, i.e. universally valid formulae differ from one class of models to another. The relation of strict inclusion between $\mathrm{BBI}_{\mathrm{ND}}$ and $\mathrm{BB} \mathrm{I}_{\mathrm{PD}}$ was, to our knowledge, an undecided proposition.

THEOREM 6.3. $\mathrm{BBI}_{\mathrm{ND}} \subsetneq \mathrm{BBI}_{\mathrm{PD}} \subsetneq \mathrm{BBI} \mathrm{T}_{\mathrm{TD}}$

Proof. The following inclusion relations TD $\subseteq \mathrm{PD} \subseteq$ ND hold between the classes of models which respectively define those three logics. Hence, only the strictness of the inclusion of validities is not obvious. This strictness is established by upcoming Theorem 6.4 and Proposition 6.5.

Consider the formula $\mathcal{I}=\neg(T * \neg)$ and a non-deterministic monoid $(M, \circ, \epsilon)$. Since $\mathcal{I}$ does not contain any variable, its Kripke interpretation does not depend on the choice of $\delta$. One can check that for any $x \in M, x \Vdash \mathcal{I}$ iff there exists $x^{\prime} \in M$ s.t. $\epsilon \in x \circ x^{\prime}$. So $\mathcal{I}$ expresses "invertibility" in Kripke semantics. The formula $(\mathcal{I} * \mathcal{I}) \rightarrow \mathcal{I}$ expresses stability of invertibility by monoidal composition.

THEOREM 6.4. With $\mathcal{I}=\neg(\top * \neg)$, the formula $(\mathcal{I} * \mathcal{I}) \rightarrow \mathcal{I}$ is valid in every partial deterministic monoid. There exists a non-deterministic monoid which is a counter-model to $(\mathcal{I} * \mathcal{I}) \rightarrow \mathcal{I}$.

Proof. First the counter-model. Consider the non-deterministic monoid $(\{\epsilon, \mathrm{x}, \mathrm{y}\}, \circ, \epsilon)$ uniquely defined by $\mathrm{x} \circ \mathrm{x}=\{\epsilon, \mathrm{y}\}, \mathrm{y} \circ \alpha=\{\mathrm{y}\}$ for any $\alpha \in\{\epsilon, \mathrm{x}, \mathrm{y}\}$ and the axioms $1 \& 2$ of Definition 2.1. ${ }^{7}$ Then $\mathrm{x} \Vdash I$ because there exists $\alpha(\alpha=\mathrm{x})$ such that $\epsilon \in \mathrm{x} \circ \alpha$. On the other hand, $\mathrm{y} \nVdash \mathcal{I}$ because there is no $\alpha$ such that $\epsilon \in \mathrm{y} \circ \alpha$ holds. So, as $\mathrm{y} \in \mathrm{x} \circ \mathrm{x}$, we have $\mathrm{y} \Vdash I * \mathcal{I}$. Thus $\mathrm{y} \nVdash(\mathcal{I} * \mathcal{I}) \rightarrow \mathcal{I}$.

Now let us prove that $(\mathcal{I} * \mathcal{I}) \rightarrow \mathcal{I}$ is valid in every partial deterministic monoid. Let $(\mathrm{M}, \circ, \epsilon)$ be a partial deterministic monoid. Let us choose $a \in \mathrm{M}$ and let us prove that $a \Vdash(\mathcal{I} * \mathcal{I}) \rightarrow \mathcal{I}$. So we suppose $a \Vdash \mathcal{I} * \mathcal{I}$ holds and we have to prove $a \Vdash \mathcal{I}$. As $a \Vdash \mathcal{I} * \mathcal{I}$, there exist $b, c \in \mathrm{M}$ such that $a \in b \circ c, b \Vdash I$ and $c \Vdash \mathcal{I}$. Thus there exist $b^{\prime}, c^{\prime} \in \mathrm{M}$ such that $\epsilon \in b \circ b^{\prime}$ and $\epsilon \in c \circ c^{\prime}$. As $\mathrm{M}$ is (partial) deterministic, we have $b \circ b^{\prime}=\{\epsilon\}, c \circ c^{\prime}=\{\epsilon\}$ and $b \circ c=\{a\}$. Thus we have $\left(b \circ b^{\prime}\right) \circ\left(c \circ c^{\prime}\right)=\{\epsilon\} \circ\{\epsilon\}=\{\epsilon\}$.

If $b^{\prime} \circ c^{\prime}=\emptyset$ then we would have $(b \circ c) \circ\left(b^{\prime} \circ c^{\prime}\right)=\{a\} \circ \emptyset=\emptyset$ but also $\left(b \circ b^{\prime}\right) \circ\left(c \circ c^{\prime}\right)=\{\epsilon\}$ and thus $\emptyset=\{\epsilon\}$ by associativity/commutativity, which is absurd. Thus $b^{\prime} \circ c^{\prime}=\left\{a^{\prime}\right\}$ and we obtain $(b \circ c) \circ\left(b^{\prime} \circ c^{\prime}\right)=\{a\} \circ\left\{a^{\prime}\right\}=a \circ a^{\prime}$ and then $a \circ a^{\prime}=\{\epsilon\}$ by associativity/commutativity. Hence, $\epsilon \in a \circ a^{\prime}$ and $a \Vdash I$.

The formula $(\neg \mid * \perp) \rightarrow I$ is inspired from the example given to establish the incompleteness of (total) monoidal Kripke semantics w.r.t. (intuitionistic) BI (see [Pym 2002] page 63).

\footnotetext{
${ }^{7}$ This non-deterministic monoid was presented in Section 2.2 as a witness that the class ND is strictly larger than PD.
} 
Proposition 6.5. The formula $(\neg \mathrm{I} * \perp) \rightarrow \mathrm{I}$ is valid in every total deterministic monoid. There exists a partial deterministic monoid which is a counter-model to $(\neg \mathrm{I} * \perp) \rightarrow \mathrm{I}$.

Proof. First the counter-model. Consider the following partial deterministic monoid $(\{\epsilon, \mathrm{x}\}, \circ, \epsilon)$ where $\mathrm{x} \circ \mathrm{x}=\emptyset$ and $\epsilon \circ \alpha=\alpha \circ \epsilon=\{\alpha\}$ for any $\alpha \in\{\epsilon, \mathrm{x}\}$. Then $\mathrm{x} \neq \epsilon$ and thus $\mathrm{x} \nVdash \mathrm{I}$. Let us prove that $\mathrm{x} \Vdash \neg l \rightarrow \perp$. Let $a, b$ such that $b \in \mathrm{x} \circ a$ and $a \Vdash \neg l$. Then $a \neq \epsilon$ and thus $a=\mathrm{x}$. Then $\mathrm{x} \circ a=\mathrm{x} \circ \mathrm{x}=\emptyset$. We get a contradiction with $b \in \mathrm{x} \circ a$. From this contradiction, we deduce $b \Vdash \perp$. Hence, $\mathrm{x} \Vdash \neg I * \perp$ and we conclude $\mathrm{x} \nVdash(\neg I * \perp) \rightarrow I$ and we have the counter-model.

Now let us prove that $(\neg \mid * \perp) \rightarrow I$ is valid in every total deterministic monoid. Let $(\mathrm{M}, \circ, \epsilon)$ be a total deterministic monoid. Let us choose $a \in \mathrm{M}$. There are two cases. Either $a=\epsilon$ or $a \neq \epsilon$. In the case $a=\epsilon$, we obviously have $a \Vdash(\neg I * \perp) \rightarrow I$. In the case $a \neq \epsilon$, let us prove $a \nVdash \neg \mid * \perp$. Suppose $a \Vdash \neg \mid * \perp$. As $a \neq \epsilon$ we have $a \Vdash \neg$. Also $a \circ a$ is not empty because $\circ$ is total. Let $b \in a \circ a$. As $a \Vdash \neg \mid \rightarrow \perp, b \in a \circ a$ and $a \Vdash \neg$, we must have $b \Vdash \perp$ which is impossible. Hence $a \nVdash \neg l * \perp$ and we conclude that $a \Vdash(\neg I \rightarrow \perp) \rightarrow I$ holds also in the case $a \neq \epsilon$.

Remark: we point out the inclusion sequences $\operatorname{ILL}_{\mathrm{ND}}^{t} \subseteq \mathrm{ILL}_{\mathrm{PD}}^{t} \subseteq \mathrm{ILL}_{\mathrm{TD}}^{t}$ as a remaining open question where $I L L_{X}^{t}$ is defined by trivial phase semantics with the monoid belonging to the class $\mathrm{X} \in\{\mathrm{ND}, \mathrm{PD}, \mathrm{TD}\}$. The question is of course: are these two inclusions strict? We remark that the counter-examples of Theorem 6.4 and Proposition 6.5 cannot be used as is because both formulae contain a negation.

\subsection{Heap models vs. free monoidal models of BBI}

In this section, we briefly explain how free monoidal models are less general that heaps model, at least with respect to BBI. The core of the argument is based on the bisimulation of multisets by heaps, a technique that was already (implicitly) used in [Brotherston and Kanovich 2010]. In Appendix C, we explicitly show how the bisimulation argument works.

Lemma 6.6. Let $\mathrm{X}$ be a set. There exists a heap monoid $\mathbb{H}_{\mathrm{L}, \mathrm{V}}$ of class $\mathrm{HM}$ and a surjective map $\varphi: \mathbb{H}_{\mathrm{L}, \mathrm{V}} \longrightarrow \mathbb{M}_{\mathrm{f}}(\mathrm{X})$ such that for any Kripke interpretation $\delta: \operatorname{Var} \longrightarrow \mathbb{P}\left(\mathbb{M}_{\mathrm{f}}(\mathrm{X})\right)$ in the free monoid $\left(\mathbb{M}_{\mathrm{f}}(\mathrm{X}), \star, \pi\right)$, the Kripke interpretation $\delta^{\prime}:$ Var $\longrightarrow \mathbb{P}\left(\mathbb{H}_{\mathrm{L}, \mathrm{V}}\right)$ in the heap monoid $\left(\mathbb{H}_{\mathrm{L}, \mathrm{V}}, \uplus, \varnothing\right)$ defined by $\delta^{\prime}=v \mapsto \varphi^{-1}(\delta(v))$ satisfies the following property:

$$
h \Vdash_{\delta^{\prime}} F \quad \text { if and only if } \varphi(h) \Vdash_{\delta} F \quad \text { for any } F \in \mathrm{BBI}
$$

Proof. The proof of this technical lemma is postponed in Appendix C.

THEOREM 6.7. The inclusion $\mathrm{BBI}_{\mathrm{HM}} \subseteq \mathrm{BBI}_{\mathrm{FM}}$ holds. Validity in heap monoids is stronger than validity in free monoids.

Proof. Let $F \in \mathrm{BBI}_{\mathrm{HM}}$ be a BBI-formula which is valid in every heap model. Then let $\left(\mathbb{M}_{\mathrm{f}}(\mathrm{X}), \star, \pi, \delta\right)$ be a Kripke interpretation in a model of class FM. Let us consider $m \in \mathbb{M}_{\mathrm{f}}(\mathrm{X})$ and let us show that $m \Vdash_{\delta} F$ holds. Let us consider $\mathbb{H}_{\mathrm{L}, \mathrm{V}}$ and $\varphi: \mathbb{H}_{\mathrm{L}, \mathrm{V}} \longrightarrow \mathbb{M}_{\mathrm{f}}(\mathrm{X})$ as obtained from Lemma 6.6. Since $\varphi$ is surjective, let us pick an $h \in \mathbb{H}_{\mathrm{L}, \mathrm{V}}$ such that $\varphi(h)=m$. Since $F \in \mathrm{BBI}_{\mathrm{HM}}$ and $\left(\mathbb{H}_{L, V}, \uplus, \varnothing, \delta^{\prime}\right)$ is a Kripke model of class HM, we deduce $h \Vdash_{\delta^{\prime}} F$. By Lemma 6.6, we conclude $m=\varphi(h) \Vdash_{\delta} F$. Thus $F$ belongs to $\mathrm{BBI}_{\mathrm{FM}}$.

Let us consider two particular models of BBI. First, the simplest heap model $\left(\mathbb{H}_{\mathbb{N},\{*\}}, \uplus, \varnothing\right)$ which is isomorphic to the partial monoid of finite subsets of $\mathbb{N}$, i.e. the partial deterministic monoid $\left(\mathbb{P}_{\mathrm{f}}(\mathbb{N}), \uplus, \emptyset\right)$. Then the free monoid over two elements which is isomorphic to the total deterministic monoid $(\mathbb{N} \times \mathbb{N},+,(0,0))$. We denote by $B_{B} I_{\mathbb{f}_{f}(\mathbb{N})}\left(\right.$ resp. BBI $\left.\left.\right|_{\mathbb{N} \times \mathbb{N}}\right)$ the set of $B B I$ formulae which are valid in every Kripke interpretation over the heap model $\left(\mathbb{P}_{\mathrm{f}}(\mathbb{N}), \uplus, \emptyset\right)$ (resp. free monoid $(\mathbb{N} \times$ $\mathbb{N},+,(0,0)))$. Then we obtain the following result:

THEOREM 6.8. The inclusion $\mathrm{BBI}_{\mathbb{P}_{\mathrm{f}}(\mathbb{N})} \subseteq \mathrm{BBI}_{\mathbb{N} \times \mathbb{N}}$ holds.

Proof. The proof is postponed in Appendix C. It is mainly a particular instance of the proofs of Lemma 6.6 and Theorem 6.7. 
Remark: the strictness of the inclusion $\left.B B\right|_{\mathbb{P}_{\mathrm{f}}(\mathbb{N})} \subseteq \mathrm{BBI}_{\mathbb{N} \times \mathbb{N}}$ remains an open question.

\section{THE UNDECIDABILITY OF BOOLEAN BI}

Having defined the Kripke semantics of BBI within the framework of non-deterministic monoids, let us explore its relations with non-deterministic trivial phase semantics for ILL.

\subsection{Trivial Phase vs. Kripke Semantics}

Let us compare the trivial phase semantic interpretation of ILL connectives and the Kripke interpretation of $\mathrm{BBI}$ connectives. Given a non-deterministic monoid $\mathcal{M}=(\mathrm{M}, \circ, \epsilon)$, a trivial phase semantic interpretation $\llbracket \cdot \rrbracket^{t}: \operatorname{Var} \mapsto \mathcal{M}^{\diamond}$ and an interpretation of variable in Kripke semantics $\delta: \operatorname{Var} \mapsto \mathbb{P}(\mathrm{M})$, we compare the trivial phase semantic interpretation of ILL-formulae and the Kripke interpretation of BBI-formulae. Recall that in trivial phase semantics all subsets of $\mathrm{M}$ are closed and thus $\mathcal{M}^{\diamond}=\mathbb{P}(\mathrm{M})$. To better compare the two semantics, we use the notation

$$
\llbracket F \rrbracket^{k}=\{m \mid m \Vdash F\}
$$

Then, using the equations defining Kripke semantics (see Section 6.1), we easily obtain the following correspondance between the interpretations of ILL and BBI connectives:

$$
\begin{aligned}
& \llbracket \perp \rrbracket^{t}=\emptyset \quad \llbracket \perp \rrbracket^{k}=\emptyset \\
& \llbracket \top]^{t}=\mathrm{M} \quad \llbracket \mathrm{\top} \rrbracket^{k}=\mathrm{M} \\
& \llbracket 1 \rrbracket]^{t}=\{\epsilon\} \quad \llbracket I \rrbracket^{k}=\{\epsilon\} \\
& \llbracket ! A \rrbracket^{t}=\{\epsilon\} \cap \llbracket A \rrbracket^{t} \\
& \llbracket \mathrm{I} \wedge A \rrbracket^{k}=\{\epsilon\} \cap \llbracket A \rrbracket^{k} \\
& \llbracket A \oplus B \rrbracket^{t}=\llbracket A \rrbracket^{t} \cup \llbracket B \rrbracket^{t} \\
& \llbracket A \vee B \rrbracket^{k}=\llbracket A \rrbracket^{k} \cup \llbracket B \rrbracket^{k} \\
& \llbracket A \& B \rrbracket^{t}=\llbracket A \rrbracket^{t} \cap \llbracket B \rrbracket^{t} \\
& \llbracket A \wedge B \rrbracket^{k}=\llbracket A \rrbracket^{k} \cap \llbracket B \rrbracket^{k} \\
& \llbracket A \otimes B \rrbracket^{t}=\llbracket A \rrbracket^{t} \circ \llbracket B \rrbracket^{t} \\
& \llbracket A * B \rrbracket^{k}=\llbracket A \rrbracket^{k} \circ \llbracket B \rrbracket^{k} \\
& \llbracket A \multimap B \rrbracket^{t}=\llbracket A \rrbracket^{t} \multimap \llbracket B \rrbracket^{t} \\
& \llbracket A * B \rrbracket^{k}=\llbracket A \rrbracket^{k} \multimap \llbracket B \rrbracket^{k}
\end{aligned}
$$

Thus, there is an obvious embedding of the connectives of ILL into BBI, which can be formalized with the following inductively defined map $(\cdot)^{\circledast}: \mathrm{ILL} \longrightarrow \mathrm{BBI}$ :

$$
\begin{aligned}
& v^{\circledast}=v \quad \text { for } v \in \operatorname{Var} \\
& \begin{array}{l}
\perp^{\circledast}=\perp \\
T^{\circledast}=\top
\end{array} \\
& 1^{\circledast}=1 \\
& (! A)^{\circledast}=\mathrm{I} \wedge A^{\circledast} \\
& (A \oplus B)^{\circledast}=A^{\circledast} \vee B^{\circledast} \\
& (A \& B)^{\circledast}=A^{\circledast} \wedge B^{\circledast} \\
& (A \otimes B)^{\circledast}=A^{\circledast} * B^{\circledast} \\
& (A \multimap B)^{\circledast}=A^{\circledast} * B^{\circledast}
\end{aligned}
$$

LEMma 7.1. If the trivial phase semantics interpretation $\llbracket \cdot \rrbracket:$ Var $\longrightarrow \mathrm{M}^{\diamond}$ and the Kripke interpretation $\delta: \operatorname{Var} \longrightarrow \mathbb{P}(\mathrm{M})$ are identical maps then the trivial phase semantics and the Kripke semantics are in the following relation:

$$
\forall F \in \mathrm{ILL}, \forall m \in \mathrm{M}, m \in \llbracket F \rrbracket \text { iff } m \Vdash F^{\circledast}
$$

Proof. Using the previous notations $\llbracket \cdot \rrbracket^{t}$ and $\llbracket \cdot \rrbracket^{k}$, we show that $\llbracket F \rrbracket^{t}=\llbracket F^{\circledast} \rrbracket^{k}$ by induction on the structure of $F$. We consider the case $F=A \otimes B$ as a typical example. Using the inductions hypotheses $\llbracket A \rrbracket^{t}=\llbracket A^{\circledast} \rrbracket^{k}$ and $\llbracket B \rrbracket^{t}=\llbracket B^{\circledast} \rrbracket^{k}$, we compute $\llbracket A \otimes B \rrbracket^{t}=\llbracket A \rrbracket^{t} \circ \llbracket B \rrbracket^{t}=\llbracket A^{\circledast} \rrbracket^{k} \circ$ $\llbracket B^{\circledast} \rrbracket^{k}=\llbracket A^{\circledast} * B^{\circledast} \rrbracket^{k}=\llbracket(A \otimes B)^{\circledast} \rrbracket^{k}$.

So if the interpretation of logical variables coincide, trivial phase semantics and Kripke semantics correspond to each other through the map $(\cdot)^{\circledast}$. Given a sequence $A_{1}, \ldots, A_{k}$ of formulae of ILL, we define $\left(A_{1}, \ldots, A_{k}\right)^{\circledast}$ by structural induction:

$$
()^{\circledast}=\mathrm{I} \quad\left(A_{1}, \ldots, A_{k+1}\right)^{\circledast}=A_{1}^{\circledast} *\left(A_{2}, \ldots, A_{k+1}\right)^{\circledast}
$$

When $\llbracket \cdot \rrbracket$ and $\delta$ are identical maps on propositional variables, it is then straightforward to prove this equivalence by induction on $k$ :

$$
m \in \llbracket A_{1} \rrbracket \circ \cdots \circ \llbracket A_{k} \rrbracket \quad \text { iff } \quad m \Vdash\left(A_{1}, \ldots, A_{k}\right)^{\circledast}
$$




\subsection{Faithfully embedding (trivial) ILL into BBI}

We define a reverse map from multisets of formulae of ILL into lists of formulae by choosing an arbitrary decidable total order among the formulae of ILL (e.g. lexicographic ordering). For any multiset $\Gamma$ of formulae of ILL, there exists a unique and computable ordered sequence of formulae $A_{1}, \ldots, A_{k}$ such that $\Gamma=\left\{A_{1}, \ldots, A_{k}\right\}$ and we define $\Gamma^{\circledast}=\left(A_{1}, \ldots, A_{k}\right)^{\circledast}$.

Proposition 7.2. The function $(\cdot)^{\circledast}:$ ILL $\longrightarrow$ BBI mapping the ILL-sequent $\Gamma \vdash C$ to the BBIformula $\Gamma^{\circledast} \rightarrow C^{\circledast}$ is a computable map from sequents of ILL to formulae of BBI.

Proof. The only thing to prove here is that the map is computable and this is done using any sorting algorithm based on the decidable total order previously chosen.

Proposition 7.3. Let $\mathcal{M}=(\mathrm{M}, \circ, \epsilon)$ be a non-deterministic monoid. Let $\Gamma \vdash C$ be a sequent of ILL. Then the sequent $\Gamma+C$ is valid in every trivial phase semantics interpretation based on $\mathcal{M}$ if and on if the formula $\Gamma^{\circledast} \rightarrow C^{\circledast}$ is valid in every Kripke interpretation based on $\mathcal{M}$.

Proof. Let us pick the ordered sequence $A_{1}, \ldots, A_{k}$ such that the identity $\Gamma=\left\lfloor A_{1}, \ldots, A_{k}\right\rfloor$ holds as a multiset equation. Let us first suppose that $A_{1}, \ldots, A_{k} \vdash C$ is valid in every trivial phase semantics interpretation based on $\mathcal{M}$. Let $\delta: \operatorname{Var} \longrightarrow \mathbb{P}(\mathrm{M})$ be a Kripke interpretation of variables in the model $\mathcal{M}$. We choose the trivial phase semantics interpretation $\llbracket \cdot \rrbracket:$ Var $\longrightarrow \mathbb{P}(\mathrm{M})$ defined by $\llbracket v \rrbracket=\delta(v)$ for any variable $v \in$ Var. By hypothesis, $A_{1}, \ldots, A_{k} \vdash C$ is valid in the interpretation $\left.\llbracket \cdot\right]$ and we deduce $\llbracket A_{1} \rrbracket \circ \cdots \circ \llbracket A_{k} \rrbracket \subseteq \llbracket C \rrbracket$. Then, by Equations (3) and (4), for any $m \in \mathrm{M}$ we have $m \Vdash\left(A_{1}, \ldots, A_{k}\right)^{\circledast} \rightarrow C^{\circledast}$. Thus the formula $\left(A_{1}, \ldots, A_{k}\right)^{\circledast} \rightarrow C^{\circledast}$ is valid in the model $(\mathrm{M}, \circ, \epsilon, \delta)$.

Now, let us suppose that $\left(A_{1}, \ldots, A_{k}\right)^{\circledast} \rightarrow C^{\circledast}$ is valid in every Kripke interpretation based on $\mathcal{M}$. Let $[\cdot]$ : Var $\longrightarrow \mathbb{P}(\mathrm{M})$ be a trivial phase semantic interpretation of variables in the model $\mathcal{M}$. We choose the Kripke interpretation $\delta: \operatorname{Var} \longrightarrow \mathbb{P}(\mathrm{M})$ defined by $\delta(v)=\llbracket v \rrbracket$ for any variable $v \in$ Var. By hypothesis, the formula $\left(A_{1}, \ldots, A_{k}\right)^{\circledast} \rightarrow C^{\circledast}$ is valid in the interpretation $\delta$ and we deduce that for any $m \in \mathrm{M}$ we have $m \Vdash\left(A_{1}, \ldots, A_{k}\right)^{\circledast} \rightarrow C^{\circledast}$. As a consequence of Equations (3) and (4), we obtain $\llbracket A_{1} \rrbracket \circ \cdots \circ \llbracket A_{k} \rrbracket \subseteq \llbracket C \rrbracket$. Hence, the sequent $A_{1}, \ldots, A_{k} \vdash C$ is valid in the trivial phase model $(\mathrm{M}, \circ, \epsilon,[[\cdot])$.

Theorem 7.4 (Embedding). Let $\mathrm{X} \in\{\mathrm{ND}, \mathrm{PD}, \mathrm{TD}, \mathrm{HM}, \mathrm{FM}\}$ be a class of non-deterministic monoids. For any sequent $\Gamma \vdash C$ of ILL, the following equivalence holds:

$$
\Gamma \vdash C \in \mathrm{ILL}_{\mathrm{X}}^{t} \quad \text { if and only if } \Gamma^{\circledast} \rightarrow C^{\circledast} \in \mathrm{BBI}_{\mathrm{X}}
$$

Proof. Obvious consequence of Proposition 7.3.

THEOREM 7.5. Trivial phase semantics restricted to heap models is complete for elLL.

Proof. Consider the inclusion sequence elLL $g=\operatorname{elLL}_{\mathrm{PD}}^{t} \subseteq \mathrm{elLL}_{\mathrm{HM}}^{t} \subseteq \mathrm{elLL}_{\mathrm{FM}}^{t}=\mathrm{elLL}_{g}$. Using Theorem 4.4, the only inclusions left to be proved are elLL $t_{\mathrm{PD}}^{t} \subseteq$ elLL ${ }_{\mathrm{HM}}^{t}$ and elLL ${ }_{\mathrm{HM}} \subseteq$ elLL $_{\mathrm{FM}}^{t}$. The first one is obvious because $\mathrm{HM} \subseteq \mathrm{PD}$ as classes of non-deterministic monoids. We prove the second inclusion. Let $\Gamma \vdash C$ be a sequent of elLL. We suppose $\Gamma \vdash C$ belongs to elLL ${ }_{\mathrm{HM}}^{t}$. Thus, it also belongs to $\mathrm{ILL}_{\mathrm{HM}}^{t}$. Then, by Theorem 7.4, we have $\Gamma^{\circledast} \rightarrow C^{\circledast} \in \mathrm{BBI}_{\mathrm{HM}}$. Thus by Theorem 6.7, we obtain $\Gamma^{\circledast} \rightarrow C^{\circledast} \in \mathrm{BBI}_{\mathrm{FM}}$. Thus by Theorem 7.4 again, we obtain $\Gamma \vdash C \in \mathrm{ILL}_{\mathrm{FM}}^{t}$. Since $\Gamma \vdash C$ is an elementary sequent, we conclude $\Gamma \vdash C \in \mathrm{elLL}_{\mathrm{FM}}^{t}$.

\subsection{The Undecidability Results}

From the preceding developments, we establish the undecidability of BBI w.r.t. Kripke semantics in any class belonging to $\{\mathrm{ND}, \mathrm{PD}, \mathrm{TD}, \mathrm{HM}, \mathrm{FM}\}$. Indeed, we have a faithful embedding from trivial ILL into BBI. But trivial ILL contains elLL as a complete and undecidable fragment. Thus the embedding transfers the undecidability to BBI.

Theorem 7.6 (Undecidability of BBI). For any class $\mathrm{X} \in\{\mathrm{ND}, \mathrm{PD}, \mathrm{TD}, \mathrm{HM}, \mathrm{FM}\}$, the set of (universally valid) formulae $\mathrm{BBI}_{\mathrm{X}}$ is not recursive. 
Proof. Suppose that there is an algorithm which decides membership in $\mathrm{BBI}_{\mathrm{X}}$. We propose the following algorithm which would then decide validity in the fragment elLL.

For a given elementary sequent $\Gamma \vdash C$ of elLL, compute the BBI formula $\Gamma^{\circledast} \rightarrow C^{\circledast}$. Decide if $\Gamma^{\circledast} \rightarrow C^{\circledast}$ belong to $\mathrm{BBI}_{\mathrm{X}}$. If true, then by Theorem 7.4, the sequent $\Gamma \vdash C$ belongs to $\mathrm{ILL}_{\mathrm{X}}^{t}$. By Theorems 4.4 and 7.5, the fragment elLL is complete w.r.t. trivial phase semantics in class $X, \Gamma \vdash C$ is a valid sequent of ILL. On the contrary, if the formula $\Gamma^{\circledast} \rightarrow C^{\circledast}$ does not belong to $\mathrm{BBI}_{\mathrm{X}}$, then by Theorem 7.4 the sequent $\Gamma \vdash C$ has a trivial phase semantics counter-model of class $\mathrm{X}$. Hence, it is an invalid sequent of ILL.

By Theorem 5.7, there is no algorithm which decide the validity of sequent of the fragment elLL. We obtain a contradiction and thus no algorithm decides membership in $\mathrm{BBI}_{\mathrm{X}}$.

THEOREM 7.7. The sets of formulae $\mathrm{BBI}_{\mathbb{P}_{\mathrm{f}}(\mathbb{N})}$ and $\mathrm{BBI}_{\mathbb{N} \times \mathbb{N}}$ are not recursive.

Proof. For $B B I_{\mathbb{N} \times \mathbb{N}}$, it is simply a consequence of the undecidability of membership in elLL ${ }_{\mathbb{N} \times \mathbb{N}}^{t}$ (see Theorem 5.8) and of Proposition 7.3. For $\left.B B\right|_{\mathbb{P}_{\mathrm{f}}(\mathbb{N})}$, let us first prove the two inclusions

$$
\mathrm{elLL}_{p} \subseteq \operatorname{elLL}_{\mathbb{P}_{\mathrm{f}}(\mathbb{N})}^{t} \subseteq \mathrm{elLL}_{\mathbb{N} \times \mathbb{N}}^{t}
$$

For elLL e $_{p} \subseteq$ elLL $_{\mathbb{P}_{\mathrm{f}}(\mathbb{N})}^{t}$, this is simply a consequence of the soundness of (trivial) phase semantics

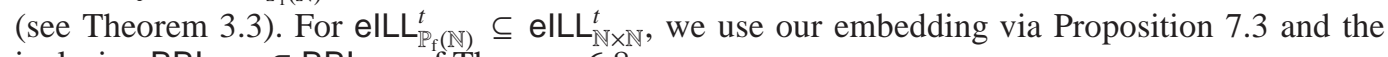
inclusion $\mathrm{BBI}_{\mathbb{P}_{\mathrm{f}}(\mathbb{N})} \subseteq \mathrm{BBI}_{\mathbb{N} \times \mathbb{N}}$ of Theorem 6.8.

Then, by Theorem 5.8, membership in the set $\operatorname{elLL}_{\mathbb{P}_{\mathrm{f}}(\mathbb{N})}^{t}$ is not decidable, and thus, by Proposition 7.3, the set $\left.B B\right|_{\mathbb{P}_{\mathrm{f}}(\mathbb{N})}$ is not recursive.

Remark: the result that $B \mathrm{BI}_{\mathbb{P}_{\mathrm{f}}(\mathbb{N})}$ is not recursive is the core result of [Brotherston and Kanovich 2010]. The indirect proof we provide here explicits the use of bisimulation to transform a model based on $\mathbb{N} \times \mathbb{N}$ into a model based on $\mathbb{P}_{\mathrm{f}}(\mathbb{N})$.

\section{CONCLUSION AND RELATED WORKS}

In this extended version of [Larchey-Wendling and Galmiche 2010], we give a full proof of the result of the undecidability of Boolean $\mathrm{BI}$ by identifying a fragment of BBI on which the semantics defined by different classes of models collapse to one. This fragment is the direct image by a faithful embedding of the elementary fragment of ILL. By studying the phase and trivial phase semantics of elLL, we establish its completeness with respect to trivial phase semantics whichever class of models is chosen amongst ND, PD, TD, HM and FM. Undecidability follows from an encoding of two counter Minsky machines computations. The faithfulness of the encoding is obtained using a trivial phase model build on the free monoid $\mathbb{N} \times \mathbb{N}$, hence we can even derive the undecidability of elLL (and later $\mathrm{BBI}$ ) restriced to the interpretations in the model $\mathbb{N} \times \mathbb{N}$.

We also bisimulate free monoids with heap monoids and thus prove that elLL is complete (and thus undecidable) for heap monoid semantics. Using a bisimulation between $\mathbb{N} \times \mathbb{N}$ and $\mathbb{P}_{\mathrm{f}}(\mathbb{N})$, we also deduce the undecidability of elLL (and thus $\mathrm{BBI}$ ) restriced to the interpretations in the model $\mathbb{P}_{\mathrm{f}}(\mathbb{N})$, which is the simplest heap model conceivable. This is basically the core result of [Brotherston and Kanovich 2010].

The question of the decidability for interpretations restricted to $\mathbb{N}$ remains open because one counter Minsky machines are a special case of pushdown automata for which accessibility is a decidable problem [Bouajjani et al. 1997].

In [Brotherston and Kanovich 2010], the authors show that undecidability also holds for Classical BI [Brotherston and Calcagno 2009] which is another variant of BI containing both an additive and a multiplicative negation. The encoding presented of [Larchey-Wendling and Galmiche 2010] which we keep in this paper would not fit for classical BI. But in [Larchey-Wendling 2010], the author proposes a modified version of our encoding which is suitable for both Boolean BI and Classical BI with a faithfulness argument based on an intepretation in the free abelian group $\mathbb{Z} \times \mathbb{Z}$. Hence he obtains another proof of undecidability suitable for both Boolean and Classical BI. 
We left remaining open problems. In particular, the classification of elLL and BBI with respect to validity in parcular classes of models, or in particular models is unfinished. Solving this requires finding elLL sequents or BBI formulae which distinguish the classes of models. This may be a difficult task which might need a better understanding of the expressive power of those two logics.

\section{A. SOUNDNESS OF NON-DETERMINISTIC PHASE SEMANTICS FOR ILL}

We recall Theorem 3.3. The proof we provide is really just an adaptation of a standard proof in Linear Logic semantics to the more general context of non-deterministic monoids.

THEorem 3.3. Let $\mathcal{M}=\left(\mathrm{M}, \circ, \epsilon,(\cdot)^{\circ}, \mathrm{K}\right)$ be a non-deterministic intuitionistic phase space and $\llbracket \cdot]:$ Var $\longrightarrow \mathcal{M}^{\diamond}$ be an interpretation of logical variables. If the sequent $A_{1}, \ldots, A_{k} \vdash B$ has a proof in S-ILL, then the inclusion $\llbracket A_{1} \rrbracket \circ \cdots \circ \llbracket A_{k} \rrbracket \subseteq \llbracket B \rrbracket$ holds.

Proof. It could be done by induction on ILL proof trees but we rather use the algebraic semantic characterization of ILL of [Troelstra 1992]. We prove that

$$
\left(\mathcal{M}^{\diamond}, \cap,(\cdot \cup \cdot)^{\diamond}, \emptyset^{\diamond},-\circ,(\cdot \circ \cdot)^{\diamond},\{\epsilon\}^{\diamond},(\mathrm{K} \cap \cdot)^{\diamond}\right)
$$

is an IL-algebra with storage operator (where $\multimap$ is defined by $\mathrm{X} \multimap \mathrm{Y}=\{k \in \mathrm{M} \mid k \circ \mathrm{X} \subseteq \mathrm{Y}\}$ ).

First, it is obvious that $\left(\mathrm{M}^{\diamond}, \cap,(\cdot \cup \cdot)^{\triangleright}, \emptyset^{\triangleright}\right)$ is a complete lattice with bottom $\emptyset^{\triangleright}$. This is the same proof as in the usual (monoidal) case because the (non-deterministic) monoidal structure does not play any role in this part of the proof. The principal argument is that $(\cdot)^{\diamond}$ is a closure operator on $\mathbb{P}(\mathrm{M})$.

Let us prove that $\left(\mathcal{M}^{\diamond},(\cdot \circ \cdot)^{\diamond},\{\epsilon\}^{\diamond}\right)$ is a commutative monoid. Obviously the set $\mathcal{M}^{\diamond}$ is stable under the operator $(\cdot \circ \cdot)^{\diamond}$ which thus induces a binary operation on $\mathcal{M}^{\diamond}$. By stability, we obtain the inclusion $\{\epsilon\}^{\diamond} \circ X^{\diamond} \subseteq(\{\epsilon\} \circ X)^{\diamond}=X^{\diamond}$ and we deduce that for any closed subset $X$ (i.e. $X=X^{\diamond}$ ), we have $\left(\{\epsilon\}^{\diamond} \circ X\right)^{\diamond} \subseteq X$. Also $X=\{\epsilon\} \circ X \subseteq\{\epsilon\}^{\diamond} \circ X \subseteq\left(\{\epsilon\}^{\diamond} \circ X\right)^{\diamond}$ by monotonicity of $\circ$ and $(\cdot)^{\diamond}$. Thus $\left(\{\epsilon\}^{\diamond} \circ X\right)^{\diamond}=X$ for any closed subset $X \in \mathcal{M}^{\diamond}$ and thus $\{\epsilon\}^{\diamond}$ is a (left) unit for $(\cdot \circ \cdot)^{\diamond}$. Then, it is obvious that $(\cdot \circ \cdot)^{\diamond}$ is a commutative operation because $\circ$ is itself commutative. We deduce that $\{\epsilon\}^{\diamond}$ is a unit for $(\cdot \circ \cdot)^{\diamond}$.

Let us prove that $(\cdot \circ \cdot)^{\diamond}$ is associative. Let $A, B, C \in \mathcal{M}^{\diamond}$. Then, by stability of $(\cdot)^{\diamond}$, we have $A \circ(B \circ C)^{\diamond} \subseteq A^{\diamond} \circ(B \circ C)^{\diamond} \subseteq(A \circ(B \circ C))^{\diamond}=(A \circ B \circ C)^{\diamond}$. Thus $\left(A \circ(B \circ C)^{\diamond}\right)^{\diamond} \subseteq(A \circ B \circ C)^{\diamond}$ holds. As $A \circ B \circ C=A \circ(B \circ C) \subseteq A \circ(B \circ C)^{\diamond} \subseteq\left(A \circ(B \circ C)^{\diamond}\right)^{\diamond}$, we deduce $(A \circ B \circ C)^{\diamond} \subseteq$ $\left(A \circ(B \circ C)^{\diamond}\right)^{\diamond}$. By double inclusion, we conclude that $(A \circ B \circ C)^{\diamond}=\left(A \circ(B \circ C)^{\diamond}\right)^{\diamond}$. Associativity of $(\cdot \circ \cdot)^{\diamond}$ follows from this last identity and associativity/commutativity of $\circ$ on $\mathbb{P}(\mathrm{M})$.

It is obvious that $(\cdot \circ \cdot)^{\diamond}$ is monotonic in both parameters because it is obtained by composition of two monotonic operators, namely $\circ$ and $(\cdot)^{\diamond}$. Let us now prove that $\multimap$ is a right-adjoint $(\cdot \circ \cdot)^{\diamond}$. First, $X \multimap Y$ is closed as soon as $Y$ is closed and $X \multimap Y^{\diamond}=X^{\diamond} \multimap Y^{\diamond}$ holds for any $X, Y \in \mathbb{P}(M)$ just as in the usual (monoidal) case. Now let $A, B, C \in \mathcal{M}^{\diamond}$. We have $(A \circ B)^{\diamond} \subseteq C$ iff $A \circ B \subseteq C$ iff $A \subseteq B \multimap C$. Thus $\multimap$ is indeed right-adjoint to $(\cdot \circ \cdot)^{\diamond}$. The fact that $\multimap$ is contra-variant w.r.t. its first operand and co-variant w.r.t. its second operand is deducible from the monotonicity of $\circ$ and the fact that $\multimap$ is right adjoint to $\circ$.

We finish by proving that $\mathrm{X} \mapsto(\mathrm{K} \cap \mathrm{X})^{\diamond}$ is a modality. First, for any $\mathrm{X} \in \mathcal{M}^{\diamond}$, as $\mathrm{K} \cap \mathrm{X} \subseteq \mathrm{X}=\mathrm{X}^{\diamond}$, we obtain $(K \cap X)^{\diamond} \subseteq X$. Then for $X, Y \in \mathcal{M}^{\diamond}$, if we suppose that $(K \cap Y)^{\diamond} \subseteq X$, then $K \cap Y \subseteq$ $\mathrm{X}$ and thus $\mathrm{K} \cap \mathrm{Y} \subseteq \mathrm{K} \cap \mathrm{X}$. Thus we obtain $(\mathrm{K} \cap \mathrm{Y})^{\diamond} \subseteq(\mathrm{K} \cap \mathrm{X})^{\diamond}$. Then, as $\epsilon \in \mathrm{K} \subseteq\{\epsilon\}^{\diamond}$, we deduce $\{\epsilon\}^{\diamond} \subseteq \mathrm{K}^{\diamond}=(\mathrm{K} \cap \mathrm{M})^{\diamond} .8$ The last condition to check is $\left((\mathrm{K} \cap \mathrm{X})^{\diamond} \circ(\mathrm{K} \cap \mathrm{Y})^{\diamond}\right)^{\diamond}=(\mathrm{K} \cap \mathrm{X} \cap \mathrm{Y})^{\diamond}$ for any $\mathrm{X}, \mathrm{Y} \in \mathcal{M}^{\diamond}$. First we have $(\mathrm{K} \cap \mathrm{X})^{\diamond} \circ(\mathrm{K} \cap \mathrm{Y})^{\diamond} \subseteq((\mathrm{K} \cap \mathrm{X}) \circ(\mathrm{K} \cap \mathrm{Y}))^{\diamond}$. As $\mathrm{K} \subseteq\{\epsilon\}^{\diamond}$, we have $(K \cap X) \circ(K \cap Y) \subseteq\{\epsilon\}^{\diamond} \circ Y \subseteq Y^{\diamond}=Y$. We also have $(K \cap X) \circ(K \cap Y) \subseteq X$. As $K \circ$ $\mathrm{K} \subseteq \mathrm{K}$ we have $(\mathrm{K} \cap \mathrm{X}) \circ(\mathrm{K} \cap \mathrm{Y}) \subseteq \mathrm{K}$ and hence, we deduce $(\mathrm{K} \cap \mathrm{X}) \circ(\mathrm{K} \cap \mathrm{Y}) \subseteq \mathrm{K} \cap \mathrm{X} \cap \mathrm{Y}$. Using stability, we compute $(\mathrm{K} \cap \mathrm{X})^{\diamond} \circ(\mathrm{K} \cap \mathrm{Y})^{\diamond} \subseteq((\mathrm{K} \cap \mathrm{X}) \circ(\mathrm{K} \cap \mathrm{Y}))^{\diamond} \subseteq(\mathrm{K} \cap \mathrm{X} \cap \mathrm{Y})^{\diamond}$ and thus $\left((\mathrm{K} \cap \mathrm{X})^{\diamond} \circ(\mathrm{K} \cap \mathrm{Y})^{\diamond}\right)^{\diamond} \subseteq(\mathrm{K} \cap \mathrm{X} \cap \mathrm{Y})^{\diamond}$. Now let us prove the reverse inclusion. Let $z \in \mathrm{K} \cap \mathrm{X} \cap \mathrm{Y}$. As

\footnotetext{
$\overline{{ }^{8} \text { Recall the identity } \emptyset^{\diamond} \multimap \emptyset^{\diamond}=\emptyset \multimap \emptyset^{\triangleright}=M}$
} 
$z \in \mathrm{K}$ then $z \in \mathrm{J}$ and we have $z \in(z \circ z)^{\diamond} \subseteq((\mathrm{K} \cap \mathrm{X}) \circ(\mathrm{K} \cap \mathrm{Y}))^{\diamond} \subseteq\left((\mathrm{K} \cap \mathrm{X})^{\diamond} \circ(\mathrm{K} \cap \mathrm{Y})^{\diamond}\right)^{\diamond}$. Hence, $\mathrm{K} \cap \mathrm{X} \cap \mathrm{Y} \subseteq\left((\mathrm{K} \cap \mathrm{X})^{\diamond} \circ(\mathrm{K} \cap \mathrm{Y})^{\diamond}\right)^{\diamond}$ and we deduce $(\mathrm{K} \cap \mathrm{X} \cap \mathrm{Y})^{\diamond} \subseteq\left((\mathrm{K} \cap \mathrm{X})^{\diamond} \circ(\mathrm{K} \cap \mathrm{Y})^{\diamond}\right)^{\diamond}$.

We can then apply Theorem 8.21 (page 80) from [Troelstra 1992]. If $A_{1}, \ldots, A_{k} \vdash B$ has a proof in ILL, then the inclusion $\llbracket A_{1}, \ldots, A_{k} \rrbracket \subseteq \llbracket B \rrbracket$ holds. It is obvious to prove that $\llbracket A_{1} \rrbracket \circ \cdots \circ \llbracket A_{k} \rrbracket \subseteq$ $\llbracket A_{1}, \ldots, A_{k} \rrbracket$ by induction on $k$ for example. So we deduce $\llbracket A_{1} \rrbracket \circ \cdots \circ \llbracket A_{k} \rrbracket \subseteq \llbracket B \rrbracket$.

\section{B. COMPLETENESS OF NON-DETERMINISTIC PHASE SEMANTICS FOR ILL}

Let Form denote the set of formulae of ILL build from Var as set of logical variables, as defined in Section 3. Let Ctx $=\mathbb{M}_{\mathrm{f}}$ (Form) denote the set of contexts build from the formulae of ILL, i.e. the set of finite multisets of ILL-formulae. Recall that a sequent is a pair $(\Gamma, C) \in \mathrm{Ctx} \times$ Form denoted $\Gamma \vdash C$

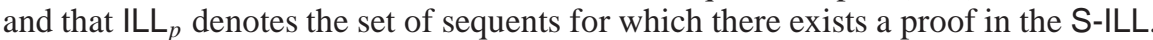

Given a set of contexts $\mathrm{X} \subseteq \mathrm{Ctx}$, a context $\Delta \in \mathrm{Ctx}$ and a formula $C \in$ Form, we denote by $\Delta, \mathrm{X} \vdash C$ the following set of sequents:

$$
\Delta, \mathrm{X} \vdash C=\{\Delta, \Gamma \vdash C \mid \Gamma \in \mathrm{X}\}
$$

We consider the following free (commutative) monoid $(\mathrm{Ctx}, \star, \pi)$ where the composition $\star$ is defined by $\Gamma \star \Delta=\{\lfloor\Gamma, \Delta\rfloor\}^{9}$ for any $\Gamma, \Delta \in \operatorname{Ctx}$ and $\pi=\lfloor\emptyset\rfloor$ is the empty context. This nondeterministic monoid ( $\mathrm{Ctx}, \star, \pi$ ) obviously belongs to the class FM. The adjoint of $\star$ is denoted $-\star$. We define the closure operator $(\cdot)^{\diamond}$ on $\mathbb{P}(\mathrm{Ctx})$ and the set $\mathrm{K} \subseteq \mathrm{Ctx}$ by

$$
\begin{aligned}
\mathrm{X}^{\diamond} & =\left\{\Gamma \in \mathrm{Ctx} \mid \forall \Delta \in \mathrm{Ctx}, \forall C \in \text { Form } \Delta, \mathrm{X} \vdash C \subseteq \mathrm{ILL}_{p} \Rightarrow \Delta, \Gamma \vdash C \in \mathrm{ILL}_{p}\right\} \\
\mathrm{K} & =\{! \Gamma \in \mathrm{Ctx} \mid \Gamma \in \mathrm{Ctx}\}
\end{aligned}
$$

Proposition B.1. (Ctx, $\left.\star, \pi,(\cdot)^{\diamond}, \mathrm{K}\right)$ is a non-deterministic phase space of class FM.

Proof. As mentioned earlier, $(\mathrm{Ctx}, \star, \pi)$ is a non-deterministic monoid of class FM. We first prove that $(\cdot)^{\diamond}$ is a stable closure, then we show that $\mathrm{K}$ verifies $\pi \in \mathrm{K} \subseteq\left\{\Gamma \in \operatorname{Ctx} \mid \Gamma \in\{\pi\}^{\diamond} \cap(\Gamma \circ \Gamma)^{\diamond}\right\}$ and $\mathrm{K} \star \mathrm{K} \subseteq \mathrm{K}$.

Let $X$ and $Y$ be two subsets of $C t x$. Let us prove $X \subseteq X$. Let $\Gamma \in X$. Then for any $\Delta, C$ we have $\{\Delta, \Gamma \vdash C\} \subseteq \Delta, \mathrm{X} \vdash C$. Hence, if $\Delta, \mathrm{X} \vdash C \subseteq \mathrm{ILL}_{p}$ holds, the property $\Delta, \Gamma \vdash C \in \mathrm{ILL}_{p}$ also holds. Thus, $\Gamma \in \mathrm{X}^{\diamond}$ holds. We have proved $\mathrm{X} \subseteq \mathrm{X}^{\diamond}$. From the definition of $(\cdot)^{\diamond}, \mathrm{X} \subseteq \mathrm{Y}$ obviously entails $\mathrm{X}^{\diamond} \subseteq \mathrm{Y}^{\diamond}$. Let us now prove that $X^{\diamond \diamond} \subseteq X^{\diamond}$. Let $\Gamma \in X^{\diamond \diamond}$ and let us prove $\Gamma \in X^{\diamond}$. We consider $\Delta, C$ such that the property $\Delta, \mathrm{X} \vdash C \subseteq \mathrm{ILL}_{p}$ holds. By definition of $(\cdot)^{\diamond}$, we deduce that $\Delta, \mathrm{X}^{\diamond} \vdash C \subseteq \mathrm{ILL}_{p}$ holds. Since $\Gamma \in \mathrm{X}^{\diamond \diamond}$, we deduce that $\Delta, \Gamma \vdash C \in \mathrm{ILL}_{p}$ holds. From $\Delta, \mathrm{X} \vdash C \subseteq \mathrm{ILL}_{p}$ we derived $\Delta, \Gamma \vdash C \in \mathrm{ILL}_{p}$, so we have proved that $\Gamma \in \mathrm{X}^{\diamond}$. Hence, $\mathrm{X}^{\diamond \diamond} \subseteq \mathrm{X}^{\diamond}$ and then $(\cdot)^{\diamond}$ is a closure operator on $\mathbb{P}(\mathrm{Ctx})$.

Let us now prove that the closure $(\cdot)^{\diamond}$ is stable, i.e. satisfies the axiom $X^{\diamond} \star Y^{\diamond} \subseteq(X \star Y)^{\diamond}$ for any two subsets $X, Y$ of $C$ tx. Since $\star$ is commutative and $(\cdot)^{\diamond}$ is a closure, it is sufficient to prove the property $X \star Y^{\diamond} \subseteq(X \star Y)^{\diamond}$ for any two subsets $X, Y$ of $C t x$ (the proof of this simplification is left to the reader). Now let us consider $\Gamma_{1} \in X$ and $\Gamma_{2} \in Y^{\diamond}$ and let us prove that $\left\lfloor\Gamma_{1}, \Gamma_{2}\right\rfloor \in(X \star Y)^{\diamond}$. So let us introduce $\Delta, C$ such that $\Delta, \mathrm{X} \star \mathrm{Y} \vdash C \subseteq \mathrm{ILL}_{p}$. Since $\Gamma_{1} \in \mathrm{X}$ holds, we deduce $\left\{\Gamma_{1}\right\} \star \mathrm{Y} \subseteq \mathrm{X} \star \mathrm{Y}$ and thus $\left\lfloor\Delta, \Gamma_{1}\right\rfloor, \mathrm{Y} \vdash C \subseteq \mathrm{ILL}_{p}$ holds. Since $\Gamma_{2} \in \mathrm{Y}^{\diamond}$ holds, we deduce $\left\lfloor\Delta, \Gamma_{1}\right\rfloor, \Gamma_{2} \vdash C \in \mathrm{ILL}_{p}$. Hence, $\Delta,\left\lfloor\Gamma_{1}, \Gamma_{2}\right\rfloor \vdash C \in \operatorname{ILL}_{p}$ holds. We conclude $\left\lfloor\Gamma_{1}, \Gamma_{2}\right\rfloor \in(\mathrm{X} \star \mathrm{Y})^{\diamond}$. We have proved that $\mathrm{X} \star \mathrm{Y}^{\diamond} \subseteq(\mathrm{X} \star \mathrm{Y})^{\diamond}$ holds for any $\mathrm{X}, \mathrm{Y} \subseteq \mathrm{Ctx}$. As a consequence, the closure $(\cdot)^{\diamond}$ is stable.

Now let us finish by checking the axioms corresponding to K. Since $\pi=\lfloor\emptyset\rfloor=\lfloor! \emptyset\rfloor$, it is obvious that $\pi \in \mathrm{K}$. Let us prove that $\mathrm{K} \subseteq\left\{\Gamma \in \mathrm{Ctx} \mid \Gamma \in\{\pi\}^{\diamond} \cap(\Gamma \star \Gamma)^{\diamond}\right\}$. Let $\Gamma \in \mathrm{K}$. There exists $\Gamma_{0}$ such that $\Gamma=! \Gamma_{0}$. Let us prove that $! \Gamma_{0} \in\{\pi\}^{\diamond}$. We consider $\Delta, C$ such that $\Delta,\{\pi\} \vdash C \subseteq \operatorname{ILL}_{p}$, which reduces to $\Delta \vdash C \in \mathrm{ILL}_{p}$. Hence $\Delta \vdash C$ has a proof in S-ILL and by multiple applications of rule $\langle\mathrm{w}\rangle$, we obtain a proof of $\Delta, ! \Gamma_{0} \vdash C$ in S-ILL. Hence $\Delta, ! \Gamma_{0} \vdash C \in \mathrm{ILL}_{p}$. We conclude that $\Gamma=! \Gamma_{0}$ belongs to $\{\pi\}^{\diamond}$. Since $\Gamma \star \Gamma=\left\{\left\lfloor! \Gamma_{0}, ! \Gamma_{0}\right\rfloor\right\}$, we prove that $! \Gamma_{0} \in\left\{\left\lfloor! \Gamma_{0}, ! \Gamma_{0}\right\rfloor\right\}^{\diamond}$ using a similar argument, replacing rule $\langle\mathrm{w}\rangle$ by rule $\langle\mathrm{c}\rangle$. We finish with a proof of $\mathrm{K} \star \mathrm{K} \subseteq \mathrm{K}$. Let $\Gamma \in \mathrm{K} \star \mathrm{K}$. By definition (1)

\footnotetext{
${ }^{9}$ Recall that $\Gamma \mapsto\lfloor\Gamma\rfloor$ is the identity map on Ctx but the extra notation $\lfloor\cdot\rfloor$ in $\{\lfloor\Gamma, \Delta\rfloor\}$ is used to here to remove the ambiguity on the denotation of the comma: here it denotes the composition of multisets, not the addition of elements in a set.
} 
of the extension of $\star$ on $\mathbb{P}(\mathrm{Ctx})$, there exists $! \Gamma_{0} \in \mathrm{K}$ and $! \Gamma_{1} \in \mathrm{K}$ such that $\Gamma \in \Gamma_{0} \star \Gamma_{1}$. We deduce $\Gamma=\left\lfloor! \Gamma_{0}, ! \Gamma_{1}\right\rfloor$ and, as a consequence $\Gamma \in \mathrm{K}$ holds.

For any formula $F$ of ILL, we denote by $\downarrow F$ the section below $F$ defined by

$$
\downarrow F=\left\{\Gamma \in \mathrm{Ctx} \mid \Gamma \vdash F \in \mathrm{ILL}_{p}\right\}
$$

It is easy to prove that sections are closed subsets of $\mathbb{P}(\mathrm{Ctx})$.

Proposition B.2. For any formula $F$ of ILL, the inclusion $(\downarrow F)^{\diamond} \subseteq \downarrow F$ holds.

Proof. For the following values of $\Delta=\lfloor\emptyset\rfloor$ and $C=F$, we obtain $\Delta, \downarrow F \vdash C \subseteq \mathrm{ILL}_{p}$. Hence, if we pick $\Gamma \in(\downarrow F)^{\diamond}$, we deduce $\Delta, \Gamma \vdash C \in \operatorname{ILL}_{p}$ by definition of $(\cdot)^{\diamond}$. We conclude $\Gamma \vdash F \in \operatorname{ILL}_{p}$ and thus $\Gamma \in \downarrow F$. Hence the inclusion $(\downarrow F)^{\diamond} \subseteq \downarrow F$ holds.

As sections are closed, it is legitimate to interpret logical variables by their section, i.e. we define the interpretation $\llbracket v \rrbracket=\downarrow v$ for every variable $v \in$ Var. The following lemma which is the core of the completeness argument was first explicited by Okada [Okada 2002] (but not for exactly the same closure operator we use here).

\section{Lemma B.3 (OKadA). For any formula $F$ of ILL, the relation $\lfloor F\rfloor \in \llbracket F \rrbracket \subseteq \downarrow F$ holds.}

Proof. The proof is done by (mutual) induction of the formula $F$. The beauty of the argument is that the semantic properties $\lfloor F\rfloor \in \llbracket F \rrbracket$ and $\llbracket F \rrbracket \subseteq \downarrow F$ correspond one to one with the rules of the $\langle$ cut $\rangle$-free S-ILL calculus.

- for a variable $v \in$ Var, the property $\lfloor v\rfloor \in \llbracket v \rrbracket$ reduces to $\lfloor v\rfloor \in \downarrow v$ which is an instance of the identity axiom $\langle$ id $\rangle$. The property $\llbracket v \rrbracket \subseteq \downarrow v$ reduces to $\downarrow v \subseteq \downarrow v$ which is trivial;

- if $F$ is a formula of type $F=A \otimes B$, then we observe that rule $\left\langle\otimes_{L}\right\rangle$ corresponds to the relation $\lfloor A \otimes B\rfloor \in\{\lfloor A, B\rfloor\}^{\diamond}$ and rule $\left\langle\otimes_{R}\right\rangle$ corresponds to the relation $\downarrow A \star \downarrow B \subseteq \downarrow A \otimes B$. Thus, using the induction hypotheses $\lfloor A\rfloor \in \llbracket A \rrbracket \subseteq \downarrow A$ and $\lfloor B\rfloor \in \llbracket B \rrbracket \subseteq \downarrow B$, we compute $\lfloor A \otimes B\rfloor \in$ $\{\lfloor A, B\rfloor\}^{\diamond} \subseteq(\lfloor A\rfloor \star\lfloor B\rfloor)^{\diamond} \subseteq(\llbracket A \rrbracket \star \llbracket B \rrbracket)^{\diamond} \subseteq \llbracket A \otimes B \rrbracket$ and $\llbracket A \otimes B \rrbracket \subseteq(\llbracket A \rrbracket \star \llbracket B \rrbracket)^{\diamond} \subseteq(\downarrow A \star \downarrow B)^{\diamond} \subseteq$ $(\downarrow A \otimes B)^{\diamond} \subseteq \downarrow A \otimes B$

- if $F=A \multimap B$, then we use the relations $\lfloor A \multimap B\rfloor \in(\downarrow A)-\star\{\lfloor B\rfloor\}^{\diamond}$ and $\{\lfloor A\rfloor\}-\star \downarrow B \subseteq \downarrow A \multimap B$ corresponding to rules $\left\langle\multimap_{L}\right\rangle$ and $\left\langle\multimap_{R}\right\rangle$ respectively. We compute $\lfloor A \multimap B\rfloor \in(\downarrow A)-\star\{\lfloor B\rfloor\}^{\diamond} \subseteq$ $\llbracket A \rrbracket \rightarrow \llbracket B \rrbracket^{\diamond}=\llbracket A \multimap B \rrbracket$ and $\llbracket A \multimap B \rrbracket=\llbracket A \rrbracket-\star \llbracket B \rrbracket \subseteq\{\lfloor A\rfloor\}-\star \downarrow B \subseteq \downarrow A \multimap B ;$

- if $F=1$, we obtain the relations $\lfloor 1\rfloor \in\{\lfloor\emptyset]\}^{\diamond}$ and $\lfloor\emptyset\rfloor \in \downarrow 1$ for rules $\left\langle 1_{L}\right\rangle$ and $\left\langle 1_{R}\right\rangle$ respectively. Thus $\lfloor 1\rfloor \in\{\lfloor\emptyset\rfloor\}^{\diamond}=\{\pi\}^{\diamond}=\llbracket 1 \rrbracket$ and $\llbracket 1 \rrbracket=\{\lfloor\emptyset\rfloor\}^{\diamond} \subseteq(\downarrow 1)^{\diamond} \subseteq \downarrow 1$;

- if $F=A \& B$, we obtain the relations $\lfloor A \& B\rfloor \in\{\lfloor A\rfloor\}^{\circ},\lfloor A \& B\rfloor \in\{\lfloor B\rfloor\}^{\circ}$ and $\downarrow A \cap \downarrow B \subseteq$ $\downarrow A \& B$ for rules $\left\langle \&_{L}^{1}\right\rangle,\left\langle \&_{L}^{2}\right\rangle$ and $\left\langle \&_{R}\right\rangle$ respectively. Thus $\lfloor A \& B\rfloor \in\{\lfloor A\rfloor\}^{\diamond} \cap\{\lfloor B\rfloor\}^{\diamond} \subseteq \llbracket A \rrbracket^{\diamond} \cap \llbracket B \rrbracket^{\diamond} \subseteq$ $\llbracket A \rrbracket \cap \llbracket B \rrbracket=\llbracket A \& B \rrbracket$ and $\llbracket A \& B \rrbracket=\llbracket A \rrbracket \cap \llbracket B \rrbracket \subseteq \downarrow A \cap \downarrow B \subseteq \downarrow A \& B$;

- if $F=\top$, we obtain the relation Ctx $\subseteq \downarrow \top$ for rule $\left\langle T_{R}\right\rangle$. Thus $\lfloor T\rfloor \in$ Ctx $=\llbracket \top \rrbracket$ and $\llbracket \top \rrbracket=\mathrm{Ctx} \subseteq \downarrow \top$;

- if $F=A \oplus B$, we obtain the relations $\lfloor A \oplus B\rfloor \in\{\lfloor A\rfloor,\lfloor B\rfloor\}^{\diamond}, \downarrow A \subseteq \downarrow A \oplus B$ and $\downarrow B \subseteq$ $\downarrow A \oplus B$ for rules $\left\langle\oplus_{L}\right\rangle,\left\langle\oplus_{R}^{1}\right\rangle$ and $\left\langle\oplus_{R}^{2}\right\rangle$ respectively. Thus $\lfloor A \oplus B\rfloor \in\{\lfloor A\rfloor,\lfloor B\rfloor\}^{\diamond}=(\{\lfloor A\rfloor\} \cup\{\lfloor B\rfloor\})^{\diamond} \subseteq$ $(\llbracket A \rrbracket \cup \llbracket B \rrbracket)^{\diamond}=\llbracket A \oplus B \rrbracket$ and $\llbracket A \oplus B \rrbracket=(\llbracket A \rrbracket \cup \llbracket B \rrbracket)^{\diamond} \subseteq(\downarrow A \cup \downarrow B)^{\diamond} \subseteq(\downarrow A \oplus B)^{\diamond} \subseteq \downarrow A \oplus B ;$

- if $F=\perp$, we obtain the relation $\lfloor\perp\rfloor \in \emptyset^{\triangleright}$ for rule $\left\langle\perp_{L}\right\rangle$. Thus $\lfloor\perp\rfloor \in \emptyset^{\triangleright}=\llbracket \perp \rrbracket$ and $\llbracket \perp \rrbracket=$ $\emptyset^{\diamond} \subseteq(\downarrow \perp)^{\diamond} \subseteq \downarrow \perp ;$

— if $F=! A$, we obtain the relations $\lfloor! A\rfloor \in\{\lfloor A\rfloor\}^{\diamond}$ and $\mathrm{K} \cap \downarrow A \subseteq \downarrow(! A)$ for rules $\left\langle!_{L}\right\rangle$ and $\left\langle!_{R}\right\rangle$ respectively. Since $\lfloor! A\rfloor \in \mathrm{K}$ by definition of $\mathrm{K}$, we deduce $\lfloor! A\rfloor \in \mathrm{K} \cap\{\lfloor A\rfloor\}^{\diamond} \subseteq \mathrm{K} \cap \llbracket A \rrbracket \subseteq \llbracket ! A \rrbracket$ and $\llbracket ! A \rrbracket=(\mathrm{K} \cap \llbracket A \rrbracket)^{\diamond} \subseteq(\mathrm{K} \cap \downarrow A)^{\diamond} \subseteq(\downarrow(! A))^{\diamond} \subseteq \downarrow(! A)$.

THEOREM 3.5. If the sequent $\Gamma \vdash A$ is valid in every free monoidal phase semantic interpretation $\left.\left(M, \circ, \epsilon,(\cdot)^{\diamond}, K,[\cdot]\right]\right)$ (i.e. with $(M, \circ, \epsilon)$ of the class $\left.\mathrm{FM}\right)$, then $\Gamma \vdash A$ has a proof in S-ILL. 
Proof. Let $A_{1}, \ldots, A_{k} \vdash B$ be a sequent which is valid in every free monoidal phase semantic interpretation. In particular, it is valid in our current interpretation $(\mathrm{Ctx}, \star, \pi)$ and we deduce that the inclusion $\llbracket A_{1} \rrbracket \star \cdots \star \llbracket A_{k} \rrbracket \subseteq \llbracket B \rrbracket$ holds. By Okada's lemma B.3, we obtain

$$
\left\lfloor A_{1}, \ldots, A_{k}\right\rfloor \in\left\lfloor A_{1}\right\rfloor \star \cdots \star\left\lfloor A_{k}\right\rfloor \subseteq \llbracket A_{1} \rrbracket \star \cdots \star \llbracket A_{k} \rrbracket \subseteq \llbracket B \rrbracket \subseteq \downarrow B
$$

and we conclude $A_{1}, \ldots, A_{k} \vdash B \in \mathrm{ILL}_{p}$. Hence, the sequent $A_{1}, \ldots, A_{k} \vdash B$ has a proof in S-ILL.

Remark: this proof does not use the cut rule $\langle$ cut $\rangle$ so it can also be used as an argument for strong completeness from which it is easy to derive a semantic proof of cut-elimination for S-ILL.

\section{BISIMULATING FREE MONOIDS WITH HEAP MONOIDS}

In this section, we give a detailed proof of Lemma 6.6. Let us fix a set X. We denote by $\left(\mathbb{M}_{\mathrm{f}}(\mathrm{X}),+, 0\right)$ the (usual) free commutative generated by $X$, i.e. $\mathbb{M}_{\mathrm{f}}(X)$ is the set of finite multisets of elements of $\mathrm{X}$. Multiset composition is denoted additively, so for example we denote by $m=\sum_{x \in \mathrm{X}} m_{x} . x$ the multiset which contains exactly $m_{x} \in \mathbb{N}$ occurrences of the variable $x$ for each $x \in \mathrm{X}$. In case $\mathrm{X}$ is infinite, it is assumed that the value of $m_{x}$ is non-zero for only a finite subset of X. Recall that there is an associated (total deterministic) free monoid of class FM which is denoted $\left(\mathbb{M}_{\mathrm{f}}(\mathrm{X})\right.$, $\left.\star, \pi\right)$ with the identities $m \star n=\{m+n\}$ and $\pi=0$.

We define the following set of locations $L=X \times \mathbb{N}$, and $L_{x}=\{x\} \times \mathbb{N}$ is a section of $L$ for each $x \in \mathrm{X}$. We also define $l_{x}^{i}=(x, i) \in \mathrm{L}$ and thus we obtain the following identities:

$$
\mathrm{L}=\biguplus_{x \in \mathrm{X}} \mathrm{L}_{x} \quad \text { and } \quad \mathrm{L}_{x}=\left\{l_{x}^{0}, l_{x}^{1}, l_{x}^{2}, \ldots\right\} \quad \text { for } x \in \mathrm{X}
$$

We define the set of values $V=\{*\}$ as a singleton set. Considering the heap monoid $\left(\mathbb{H}_{L, V}, \sqcup, \varnothing\right)$, we define a map $\varphi: \mathbb{H}_{\mathrm{L}, \mathrm{V}} \longrightarrow \mathbb{M}_{\mathrm{f}}(\mathrm{X})$ by

$$
\varphi(h)=\sum_{x \in \mathrm{X}} \operatorname{card}\left(\operatorname{def}(h) \cap \mathrm{L}_{x}\right) \cdot x
$$

Proposition C.1. The map $\varphi: \mathbb{H}_{\mathrm{L}, \mathrm{V}} \longrightarrow \mathbb{M}_{\mathrm{f}}(\mathrm{X})$ satisfies the following properties:

(1) $\varphi$ is a surjective map;

(2) if $m_{1}, m_{2} \in \mathbb{M}_{\mathrm{f}}(\mathrm{X})$ and $h \in \mathbb{H}_{\mathrm{L}, \mathrm{V}}$ satisfy $\varphi(h)=m_{1}+m_{2}$ then there exists $h_{1}, h_{2} \in \mathbb{H}_{\mathrm{L}, \mathrm{V}}$ such that $\varphi\left(h_{1}\right)=m_{1}, \varphi\left(h_{2}\right)=m_{2}$ and $h_{1} \uplus h_{2}=\{h\}$;

(3) for any $m_{1} \in \mathbb{M}_{\mathrm{f}}(\mathrm{X})$ and any $h_{2} \in \mathbb{H}_{\mathrm{L}, \mathrm{V}}$ there exists $h_{1} \in \mathbb{H}_{\mathrm{L}, \mathrm{V}}$ such that $\operatorname{def}\left(h_{1}\right) \cap \operatorname{def}\left(h_{2}\right)=\emptyset$ and $\varphi\left(h_{1}\right)=m_{1}$;

(4) $\varphi\left(h_{1} \uplus h_{2}\right)=\varphi\left(h_{1}\right) \star \varphi\left(h_{2}\right)$ when $\operatorname{def}\left(h_{1}\right) \cap \operatorname{def}\left(h_{2}\right)=\emptyset$;

(5) $\varphi(h)=0$ if and only if $h=\varnothing$ for any $h \in \mathbb{H}_{\mathrm{L}, \mathrm{V}}$;

(6) $\varphi^{-1}(\mathrm{~A}) \uplus \varphi^{-1}(\mathrm{~B})=\varphi^{-1}(\mathrm{~A} \star \mathrm{B})$ for any $\mathrm{A}, \mathrm{B} \subseteq \mathbb{M}_{\mathrm{f}}(\mathrm{X})$.

Proof. Let us prove Property (1) and show that $\varphi$ is a surjective map. Let $m=\sum_{x \in \mathrm{X}} m_{x} . x$ be a finite multiset. Then the set $\left\{\left(l_{x}^{i}, *\right) \mid 0 \leqslant i<m_{x}\right\}$ is the graph of a partial function and we denote this function by $h_{m}$. It can be easily be checked that $\operatorname{def}\left(h_{m}\right)$ is a finite subset of $\mathrm{L}$ and that

$$
\varphi\left(h_{m}\right)=\sum_{x \in \mathrm{X}} \operatorname{card}\left\{l_{x}^{i} \mid 0 \leqslant i<m_{x}\right\} \cdot x=\sum_{x \in \mathrm{X}} m_{x} \cdot x=m
$$

Let use prove Property (2). Let $m, n \in \mathbb{M}_{\mathrm{f}}(\mathrm{X})$ and $h \in \mathbb{H}_{\mathrm{L}, \mathrm{V}}$ be such that $\varphi(h)=m+n$. For each $x \in \mathrm{X}$, we have card( $\left.\operatorname{def}(h) \cap \mathrm{L}_{x}\right)=m_{x}+n_{x}$. Let us partition $\operatorname{def}(h) \cap \mathrm{L}_{x} \operatorname{in} \operatorname{def}(h) \cap \mathrm{L}_{x}=\mathrm{L}_{x}^{1} \cup \mathrm{L}_{x}^{2}$ such that card $\left(\mathrm{L}_{x}^{1}\right)=m_{x}$ and $\operatorname{card}\left(\mathrm{L}_{x}^{2}\right)=n_{x}$. Then let $h_{1}$ (resp. $\left.h_{2}\right)$ be the partial function with graph $\left\{\left(l_{x}^{i}, *\right) \mid l_{x}^{i} \in \mathrm{L}_{x}^{1}\right\}$ (resp. $\left.\left\{\left(l_{x}^{i}, *\right) \mid l_{x}^{i} \in \mathrm{L}_{x}^{2}\right\}\right)$. The reader can check that $\varphi\left(h_{1}\right)=m_{1}, \varphi\left(h_{2}\right)=m_{2}$ and $h_{1} \uplus h_{2}=\{h\}$ hold.

Let us prove Property (3). Let us write $m_{1}=\sum_{x \in \mathrm{X}} m_{x}^{1} . x$. For $x \in \operatorname{Var}$, since $\mathrm{L}_{x} \backslash \operatorname{def}\left(h_{2}\right)$ is an infinite set, let us choose $\mathrm{L}_{x}^{1}$ such that $\mathrm{L}_{x}^{1} \subseteq \mathrm{L}_{x} \backslash \operatorname{def}\left(h_{2}\right)$ and card $\left(\mathrm{L}_{x}^{1}\right)=m_{x}^{1}$. Now let us consider 
the partial function $h_{1}$ defined by the graph $\left\{\left(l_{x}^{i}, *\right) \mid l_{x}^{i} \in \mathrm{L}_{x}^{1}\right\}$. It is obvious that $\operatorname{def}\left(h_{1}\right)$ is finite, $\operatorname{def}\left(h_{1}\right) \cap \operatorname{def}\left(h_{2}\right)=\emptyset$ and $\varphi\left(h_{1}\right)=m_{1}$.

Let us prove Property (4). Let $h_{1}, h_{2} \in \mathbb{H}_{\mathrm{L}, \mathrm{V}}$ such that $\operatorname{def}\left(h_{1}\right) \cap \operatorname{def}\left(h_{2}\right)=\emptyset$. Let $h$ be the result of the composition of $h_{1}$ and $h_{2}$, i.e. $h_{1} \uplus h_{2}=\{h\}$. Then card $\left(\operatorname{def}(h) \cap \mathrm{L}_{x}\right)=\operatorname{card}\left(\operatorname{def}\left(h_{1}\right) \cap \mathrm{L}_{x}\right)+$ $\operatorname{card}\left(\operatorname{def}\left(h_{1}\right) \cap \mathrm{L}_{x}\right)$ and we deduce $\varphi(h)=\varphi\left(h_{1}\right)+\varphi\left(h_{2}\right)$, hence $\varphi\left(h_{1} \uplus h_{2}\right)=\left\{\varphi\left(h_{1}\right)+\varphi\left(h_{2}\right)\right\}$.

Property (5) is obvious. Let us prove Property (6). First let us consider the inclusion $\varphi^{-1}(A \star B) \subseteq$ $\varphi^{-1}(\mathrm{~A}) \uplus \varphi^{-1}(\mathrm{~B})$. Let us pick $h \in \varphi^{-1}(\mathrm{~A} \star \mathrm{B})$. Then $\varphi(h) \in \mathrm{A} \star \mathrm{B}$ so there exists $m_{1} \in \mathrm{A}$ and $m_{2} \in \mathrm{B}$ such that $\varphi(h)=m_{1}+m_{2}$. By Property (2), there exists $h_{1}, h_{2}$ such that $\varphi\left(h_{1}\right)=m_{1}, \varphi\left(h_{2}\right)=m_{2}$ and $h_{1} \uplus h_{2}=\{h\}$. Hence $h_{1} \in \varphi^{-1}(\mathrm{~A})$ and $h_{2} \in \varphi^{-1}(\mathrm{~B})$. As $h_{1} \uplus h_{2}=\{h\}$, we get $h \in \varphi^{-1}(\mathrm{~A}) \uplus \varphi^{-1}(\mathrm{~B})$. Let us consider the reverse inclusion $\varphi^{-1}(\mathrm{~A}) \uplus \varphi^{-1}(\mathrm{~B}) \subseteq \varphi^{-1}(\mathrm{~A} \star \mathrm{B})$. Let $h \in \varphi^{-1}(\mathrm{~A}) \uplus \varphi^{-1}(\mathrm{~B})$. Then there exists $h_{1} \in \varphi^{-1}(\mathrm{~A})$ and $h_{2} \in \varphi^{-1}(\mathrm{~B})$ such that $h \in h_{1} \uplus h_{2}$. Then we have $\operatorname{def}\left(h_{1}\right) \cap \operatorname{def}\left(h_{2}\right)=\emptyset$ (otherwise $h_{1} \uplus h_{2}=\emptyset$ ) and by Property (4), we deduce $\varphi(h)=\varphi\left(h_{1}\right)+\varphi\left(h_{2}\right) \in \mathrm{A} \star \mathrm{B}$. Hence $h \in \varphi^{-1}(\mathrm{~A} \star \mathrm{B})$.

Lemma C.2 (Bisimulation). Let $R_{\varphi} \subseteq \mathbb{H}_{\mathrm{L}, \mathrm{V}} \times \mathbb{M}_{\mathrm{f}}(\mathrm{X})$ be the binary relation defined by the graph of $\varphi$, i.e. $h R_{\varphi} m$ iff $\varphi(h)=m$. Then $R_{\varphi}$ is a bisimulation between non-deterministic monoids, i.e. it satisfies the following property for any $h \in \mathbb{H}_{\mathrm{L}, \mathrm{V}}$ and any $m \in \mathbb{M}_{\mathrm{f}}(\mathrm{X})$

$$
h R_{\varphi} m \Rightarrow\left\{\begin{array}{l}
h=\varnothing \text { iff } m=\pi \\
\forall h_{1}, h_{2} h \in h_{1} \uplus h_{2} \Rightarrow \exists m_{1}, m_{2} m \in m_{1} \star m_{2} \text { and } h_{1} R_{\varphi} m_{1} \text { and } h_{2} R_{\varphi} m_{2} \\
\forall m_{1}, m_{2} m \in m_{1} \star m_{2} \Rightarrow \exists h_{1}, h_{2} h \in h_{1} \uplus h_{2} \text { and } h_{1} R_{\varphi} m_{1} \text { and } h_{2} R_{\varphi} m_{2} \\
\forall h_{1}, h_{2} h_{2} \in h_{1} \uplus h \Rightarrow \exists m_{1}, m_{2} m_{2} \in m_{1} \star m \text { and } h_{1} R_{\varphi} m_{1} \text { and } h_{2} R_{\varphi} m_{2} \\
\forall m_{1}, m_{2} m_{2} \in m_{1} \star m \Rightarrow \exists h_{1}, h_{2} h_{2} \in h_{1} \uplus h \text { and } h_{1} R_{\varphi} m_{1} \text { and } h_{2} R_{\varphi} m_{2}
\end{array}\right.
$$

Proof. Let us first prove that $h R_{\varphi} m \Rightarrow(h=\varnothing$ iff $m=\pi)$. Let $h$ and $m$ such that $h R_{\varphi} m$ holds. Then by definition, we obtain $\varphi(h)=m$. If $m=\pi(=0)$, then by Property (5) of Proposition C.1, we obtain $h=\varnothing$ and thus $(h, m) \in\{(\varnothing, \pi)\}$. If $m \neq 0$ then by Property (5) of Proposition C.1, we obtain $h \neq \varnothing$ and thus $(h, m) \in \mathbb{M}_{\mathrm{f}}(\mathrm{X}) \backslash\{\pi\}$.

Let us now prove the four co-induction properties. Let $h$ and $m$ such that $h R_{\varphi} m$ holds. Then $\varphi(h)=m$ holds.

- Let $h_{1}, h_{2} \in \mathbb{H}_{\mathrm{L}, \mathrm{V}}$ such that $h \in h_{1} \uplus h_{2}$. Let $m_{1}=\varphi\left(h_{1}\right)$ and $m_{2}=\varphi\left(h_{2}\right)$. By Property (4) of Proposition C.1, we obtain $m=\varphi(h) \in \varphi\left(h_{1}\right) \star \varphi\left(h_{2}\right)=m_{1} \star m_{2}, h_{1} R_{\varphi} m_{1}$ and $h_{2} R_{\varphi} m 2$;

Let $m_{1}, m_{2} \in \mathbb{M}_{\mathrm{f}}(\mathbf{X})$ such that $m=m_{1}+m_{2}$. Property (2) of Proposition C.1, there exists $h_{1}, h_{2} \in \mathbb{H}_{\mathrm{L}, \mathrm{V}}$ such that $\varphi\left(h_{1}\right)=m_{1}, \varphi\left(h_{2}\right)=m_{2}$ and $h_{1} \uplus h_{2}=\{h\}$. Hence, $h \in h_{1} \uplus h_{2}, h_{1} R_{\varphi} m_{1}$ and $h_{2} R_{\varphi} m 2$

- Let $h_{1}, h_{2} \in \mathbb{H}_{\mathrm{L}, \mathrm{V}}$ such that $h_{2} \in h_{1} \uplus h$. Let $m_{1}=\varphi\left(h_{1}\right)$ and $m_{2}=\varphi\left(h_{2}\right)$. By Property (4) of Proposition C.1, we obtain $m_{2}=\varphi\left(h_{2}\right) \in \varphi\left(h_{1}\right) \star \varphi(h)=m_{1} \star m, h_{1} R_{\varphi} m_{1}$ and $h_{2} R_{\varphi} m 2$;

- Let $m_{1}, m_{2} \in \mathbb{M}_{\mathrm{f}}(\mathrm{X})$ such that $m_{2}=m_{1}+m$. By Property (3) of Proposition C.1, let us choose $h_{1}$ such that $\operatorname{def}\left(h_{1}\right) \cap \operatorname{def}(h)=\emptyset$ and $\varphi\left(h_{1}\right)=m_{1}$. Hence, $h_{1} R_{\varphi} m_{1}$ holds. Since $\operatorname{def}\left(h_{1}\right) \cap \operatorname{def}(h)=\emptyset$, let $h_{2}$ be the unique heap such that $h_{2} \in h_{1} \uplus h$. By Property (4) of Proposition C.1, we obtain $\varphi\left(h_{2}\right)=\varphi\left(h_{1}\right)+\varphi(h)=m_{1}+m=m_{2}$. Hence $h_{2} R_{\varphi} m_{2}$ holds.

LEMmA 6.6. Let $\mathrm{X}$ be a set. There exists a heap monoid $\mathbb{H}_{\mathrm{L}, \mathrm{V}}$ of class $\mathrm{HM}$ and a surjective map $\varphi: \mathbb{H}_{\mathrm{L}, \mathrm{V}} \longrightarrow \mathbb{M}_{\mathrm{f}}(\mathrm{X})$ such that for any Kripke interpretation $\delta: \operatorname{Var} \longrightarrow \mathbb{P}\left(\mathbb{M}_{\mathrm{f}}(\mathrm{X})\right)$ in the free monoid $\left(\mathbb{M}_{\mathrm{f}}(\mathrm{X}), \star, \pi\right)$, the Kripke interpretation $\delta^{\prime}: \operatorname{Var} \longrightarrow \mathbb{P}\left(\mathbb{H}_{\mathrm{L}, \mathrm{V}}\right)$ in the heap monoid $\left(\mathbb{H}_{\mathrm{L}, \mathrm{V}}, \uplus, \varnothing\right)$ defined by $\delta^{\prime}=v \mapsto \varphi^{-1}(\delta(v))$ satisfies the following property:

$$
h \Vdash_{\delta^{\prime}} F \text { if and only if } \varphi(h) \Vdash_{\delta} F \quad \text { for any } F \in \mathrm{BBI}
$$

Proof. By induction on the structure of $F$, we prove the following property:

$$
\forall h, m \quad h R_{\varphi} m \Rightarrow\left(h \Vdash_{\delta^{\prime}} F \text { iff } m \Vdash_{\delta} F\right)
$$

Let us proceed by case analysis on the structure of $F$ : 
- if $F$ is reduced to a logical variable $v \in$ Var, then $h R_{\varphi} m$ implies $\varphi(h)=m$ and thus we compute: $h \Vdash_{\delta^{\prime}} v$ iff $h \in \delta^{\prime}(v)$ iff $h \in \varphi^{-1}(\delta(v))$ iff $\varphi(h) \in \delta(v)$ iff $m \in \delta(v)$ iff $m \Vdash_{\delta} v$;

- if $F$ is the multiplicative unit I then the relation reduces to $h R_{\varphi} m \Rightarrow(h=\varnothing$ iff $m=\pi)$ which is a consequence of Lemma C.2;

- if $F=A * B$, let us suppose $h \Vdash_{\delta^{\prime}} A * B$ and let us prove $m \Vdash_{\delta} A * B$. By definition of Kripke semantics, there exists $h_{1}, h_{2}$ such that $h \in h_{1} \uplus h_{2}, h_{1} \Vdash_{\delta^{\prime}} A$ and $h_{2} \Vdash_{\delta^{\prime}} B$. Since $h R_{\varphi} m$, by Lemma C.2, we obtain $m_{1}, m_{2}$ such that $m \in m_{1} \star m_{2}, h_{1} R_{\varphi} m_{1}$ and $h_{2} R_{\varphi} m_{2}$. By induction, we get $m_{1} \Vdash_{\delta} A$ and $m_{2} \Vdash_{\delta} B$. Hence, by definition of Kripke semantics, we deduce $m \Vdash_{\delta} A * B$. We proceed in a perfectly symetric way for $m \Vdash_{\delta} A * B \Rightarrow h \Vdash_{\delta^{\prime}} A * B$;

- if $F=A * B$, let us suppose $h \Vdash_{\delta^{\prime}} A * B$ and let us prove $m \Vdash_{\delta} A * B$. So let us $m_{1}, m_{2}$ consider such that $m_{2} \in m_{1} \star m$ and $m_{1} \Vdash_{\delta} A$ and let us prove that $m_{2} \Vdash_{\delta} B$. Since $h R_{\varphi} m$, by Lemma C.2, we obtain $h_{1}, h_{2}$ such that $h_{2} \in h_{1} \uplus h, h_{1} R_{\varphi} m_{1}$ and $h_{2} R_{\varphi} m_{2}$. By induction, we get $h_{1} \Vdash_{\delta^{\prime}} A$. Hence, by definition of Kripke semantics for $*$, we deduce $h_{2} \Vdash_{\delta^{\prime}} B$. By induction again, we derive $m_{2} \Vdash_{\delta} B$. Symmetrically we obtain $m \Vdash_{\delta} A * B \Rightarrow h \Vdash_{\delta^{\prime}} A * B$;

- if the outermost connective of $F$ is not multiplicative, i.e. belongs to $\{\perp, \top, \neg, \vee, \wedge, \rightarrow\}$, then the equivalence is trivially obtained from the induction hypothesis because of the pointwise definition of the Kripke semantics of non-linear connectives.

Let us consider the following map between the heap monoid $\left(\mathbb{P}_{\mathrm{f}}(\mathbb{N}), \uplus, \emptyset\right)$ and the free monoid $(\mathbb{N} \times \mathbb{N},+,(0,0))$. Let use split $\mathbb{N}$ into two infinite parts such as $\mathbb{N}=\mathbb{E} \uplus \mathbb{O}$ where $\mathbb{O}=\{2 n+1 \mid n \in \mathbb{N}\}$ and $\mathbb{E}=\{2 n \mid n \in \mathbb{N}\}$. We define the $\operatorname{map} \psi: \mathbb{P}_{\mathrm{f}}(\mathbb{N}) \longrightarrow \mathbb{N} \times \mathbb{N}$ by

$$
\psi(\mathrm{K})=(\operatorname{card}(\mathrm{K} \cap \mathbb{E}), \operatorname{card}(\mathrm{K} \cap \mathbb{O})) \quad \text { for } \mathrm{K} \text { finite subset of } \mathbb{N}
$$

The map $\psi$ is just a particular case of the map $\varphi$ where the set $X$ has two elements like for example $X=\{0,1\}$. Then the map $\psi$ is surjective and the binary relation defined by its graph is a bisimulation between non-deterministic monoids as defined in Lemma C.2. Hence, we imediatly derive the following result:

Lemma C.3. There exists a surjective map $\psi: \mathbb{P}_{\mathrm{f}}(\mathbb{N}) \longrightarrow \mathbb{N} \times \mathbb{N}$ such that for any Kripke interpretation $\delta: \operatorname{Var} \longrightarrow \mathbb{P}(\mathbb{N} \times \mathbb{N})$ in the free monoid $(\mathbb{N} \times \mathbb{N},+,(0,0))$, the Kripke interpretation $\delta^{\prime}: \operatorname{Var} \longrightarrow \mathbb{P}\left(\mathbb{P}_{\mathrm{f}}(\mathbb{N})\right)$ in the heap monoid $\left(\mathbb{P}_{\mathrm{f}}(\mathbb{N}), \uplus, \emptyset\right)$ defined by $\delta^{\prime}=v \mapsto \psi^{-1}(\delta(v))$ satisfies the following property:

$$
h \Vdash_{\delta^{\prime}} F \quad \text { if and only if } \quad \psi(h) \Vdash_{\delta} F \quad \text { for any } F \in \mathrm{BBI}
$$

We deduce a proof of Theorem 6.8

THEOREM 6.8. The inclusion $\mathrm{BBI}_{\mathbb{P}_{\mathrm{f}}(\mathbb{N})} \subseteq \mathrm{BBI}_{\mathbb{N} \times \mathbb{N}}$ holds.

Proof. Let $F \in \mathrm{BBI}_{\mathbb{P}_{\mathrm{f}}(\mathbb{N})}$ be a BBI-formula which is valid in the heap model $\left(\mathbb{P}_{\mathrm{f}}(\mathbb{N}), \biguplus, \emptyset\right)$. Then let $\delta: \operatorname{Var} \longrightarrow \mathbb{P}(\mathbb{N} \times \mathbb{N})$ be a Kripke interpretation in the free monoid $(\mathbb{N} \times \mathbb{N},+,(0,0))$. Let us consider $(a, b) \in \mathbb{N} \times \mathbb{N}$ and let us show that $(a, b) \Vdash_{\delta} F$ holds. Since $\psi$ is surjective, let us pick $\mathrm{K} \in \mathbb{P}_{\mathrm{f}}(\mathbb{N})$ such that $\psi(\mathrm{K})=(a, b)$ (for instance, $\mathrm{K}=\{0, \ldots, 2 a-2\} \cup\{1, \ldots, 2 b-1\}$ would fit). Since $F \in \mathrm{BBI}_{\mathbb{P}_{\mathrm{f}}(\mathbb{N})}$, we deduce $\mathrm{K} \Vdash_{\delta^{\prime}} F$. By Lemma C.3, we conclude $(a, b)=\psi(\mathrm{K}) \Vdash_{\delta} F$. Thus $F$ belongs to $B B I_{\mathbb{N} \times \mathbb{N}}$.

\section{REFERENCES}

Bouajuani, A., Esparza, J., And Maler, O. 1997. Reachability Analysis of Pushdown Automata: Application to ModelChecking. In CONCUR'97: Concurrency Theory, 8th International Conference, Warsaw, Poland. Lecture Notes in Computer Science Series, vol. 1243. Springer, 135-150.

Brotherston, J. 2010. A Unified Display Proof Theory for Bunched Logic. Electronic Notes in Theoretical Computer Science $265,197-211$. 
Brotherston, J. and Calcagno, C. 2009. Classical BI: a logic for reasoning about dualising resources. In Proceedings of the 36th ACM SIGPLAN-SIGACT Symposium on Principles of Programming Languages, Savannah, GA, USA. ACM, 328-339.

Brotherston, J. and Kanovich, M. I. 2010. Undecidability of Propositional Separation Logic and Its Neighbours. In Proceedings of the 25th Annual IEEE Symposium on Logic in Computer Science, Edinburgh, United Kingdom. IEEE Computer Society, 130-139.

Calcagno, C., Cardelli, L., and Gordon, A. 2005. Deciding Validity in a Spatial Logic for Trees. Journal of Functional Programming 15, 4, 543-572.

de Groote, P., Guillaume, B., and Salvati, S. 2004. Vector addition tree automata. In Proceedings of the 19th IEEE Symposium on Logic in Computer Science, Turku, Finland. IEEE Computer Society, 64-73.

Galmiche, D. and LarcheY-Wenduing, D. 2006. Expressivity properties of Boolean BI through relational models. In FSTTCS 2006: Foundations of Software Technology and Theoretical Computer Science, 26th International Conference, Kolkata, India. Lecture Notes in Computer Science Series, vol. 4337. Springer, 357-368.

Galmiche, D., Méry, D., And Pym, D. 2005. The semantics of BI and resource tableaux. Mathematical Structures in Computer Science 15, 6, 1033-1088.

Ghilardi, S. and Meloni, G. 1990. Modal logics with n-ary connectives. Zeitschr. f. math. Logik und Grundlagen d. Math 36, 193-215.

GiRARD, J.-Y. 1987. Linear logic. Theoretical Computer Science 50, 1, 1-102.

IshtiaQ, S. AND O'Hearn, P. 2001. BI as an Assertion Language for Mutable Data Structures. In Proceedings of the 28th ACM SIGPLAN-SIGACT Symposium on Principles of Programming languages, London, England. ACM, 14-26.

Kanovich, M. 1994. Linear Logic as a Logic of Computations. Annals of Pure and Applied Logic 67, 1-3, $183-212$.

Kanovich, M. 1995. The direct simulation of Minsky machines in linear logic. In Advances in Linear Logic, J.-Y. Girard, Y. Lafont, and L. Regnier, Eds. London Mathematical Society Lecture Note Series, vol. 222. Cambridge University Press, Chapter 2, 123-145.

LAFont, Y. 1996. The undecidability of second order linear logic without exponentials. Journal of Symbolic Logic 61, 2 , $541-548$.

LAFont, Y. AND ScEdrov, A. 1996. The undecidability of second order multiplicative linear logic. Information and Computation 125, 1, 46-51.

Larchey-Wendling, D. 2010. An Alternative Direct Simulation of Minsky Machines into Classical Bunched Logics via Group Semantics. Electronic Notes in Theoretical Computer Science 265, 369-387.

Larchey-Wendling, D. and Galmiche, D. 2009. Exploring the relation between Intuitionistic BI and Boolean BI: an unexpected embedding. Mathematical Structures in Computer Science 19, 3, 435-500.

Larchey-Wendling, D. and Galmiche, D. 2010. The Undecidability of Boolean BI through Phase Semantics. In Proceedings of the 25th Annual IEEE Symposium on Logic in Computer Science, Edinburgh, United Kingdom. IEEE Computer Society, 140-149.

Minsky, M. 1961. Recursive unsolvability of Post's problem of 'tag' and other topics in the theory of Turing machines. Annals of Mathematics 74, 3, 437-455.

O'Hearn, P. And Pym, D. 1999. The Logic of Bunched Implications. Bulletin of Symbolic Logic 5, 2, $215-244$.

ОкаDA, M. 2002. A uniform semantic proof for cut-elimination and completeness of various first and higher order logics. Theoretical Computer Science 281, 1-2, 471-498.

PyM, D. 2002. The Semantics and Proof Theory of the Logic of Bunched Implications. Applied Logic Series Series, vol. 26. Kluwer Academic Publishers. Errata available at http://www.cs.ac.uk/ pym/pym-tofts-fac-errata.pdf.

Troelstra, A. 1992. Lectures on Linear Logic. Lecture Notes Series, vol. 29. CSLI, Stanford, California.

YetTer, D. 1990. Quantales and (Noncommutative) Linear Logic. Journal of Symbolic Logic 55, 1, 41-64. 